\title{
Stochastic modeling in sediment dynamics: Exner equation for planar bed incipient bed load transport conditions
}

\author{
Christophe Ancey ${ }^{1}$ \\ Received 13 January 2009; revised 18 August 2009; accepted 7 December 2009; published 29 April 2010.
}

[1] Even under flow equilibrium conditions, river bed topography continuously evolves with time, producing trains of irregular bed forms. The idea has recently emerged that the variability in the bed form geometry results from some randomness in sediment flux. In this paper, we address this issue by using the Exner equation and a population exchange model derived in an earlier paper. In this model, particle entrainment and deposition are idealized as population exchanges between the stream and the bed, which makes it possible to use birth-death Markov process theory to track the number of moving grains. The paper focuses on nascent bed forms on initially planar beds, a situation in which the coupling between the stream and bed is weak. In a steady state, the number of moving particles follows a negative binomial distribution. Although this probability distribution does not enter the family of heavy-tailed distributions, it may give rise to large and frequent fluctuations because the standard deviation can be much larger than the mean, a feature that is not accounted for with classic probability laws (e.g., Hamamori's law) used so far for describing bed load fluctuations. In the large-system limit, the master equation of the birth-death Markov process can be transformed into a Fokker-Planck equation. This transformation is used here to show that the number of moving particles can be described as an Ornstein-Uhlenbeck process. An important consequence is that in the long term, the number of moving particles follows a Gaussian distribution. Laboratory experiments show that this approximation is correct when the mean number per unit length of stream, $\bar{N} / L$, is sufficiently large (typically two particles per centimeter in our experiments). The particle number fluctuations give rise to bed elevation fluctuations, whose spectrum falls off like $\omega^{-2}$ in the high-frequency regime (with $\omega$ the angular frequency) and variance grows linearly with time. These features are in agreement with recent observations on bed form development (in particular, ripple growth).

Citation: Ancey, C. (2010), Stochastic modeling in sediment dynamics: Exner equation for planar bed incipient bed load transport conditions, J. Geophys. Res., 115, F00A11, doi:10.1029/2009JF001260.

\section{Introduction}

[2] The Exner equation has attracted considerable attention in recent years. This equation expresses the mass balance of the bed by stating that the local rate of change in the bed elevation is related to the spatial gradient of the solid discharge (see (14) below). The equation was termed in honor of Felix Exner, an Austrian physicist, who notably investigated the development of dunes in sand bed rivers. In his seminal paper, Exner [1925] assumed that the erosion/ deposition rate is proportional to the streamwise gradient of the flow velocity. With this assumption, he showed that the mass balance equation for the bed takes the form of a nonlinear hyperbolic advection equation, which accounts for

\footnotetext{
${ }^{1}$ School of Architecture, Civil and Environmental Engineering, École Polytechnique Fédérale de Lausanne, Lausanne, Switzerland.

Copyright 2010 by the American Geophysical Union. 0148-0227/10/2009JF001260
}

formation and migration of bed forms [see also Kubatko and Westerink, 2007].

[3] Much of the early work has considered bed load transport as a continuous process, i.e., a process that can be conveniently described within the framework of continuum mechanics in terms of relations between mean values. However, field and laboratory observations have shown that bed load transport exhibits considerable variability in time and space, in particular at low flow rates [Ergenzinger, 1988]. Field surveys have shown that bed load transport time series are highly fluctuating signals, which may be intermittent and comprise pulses that are more or less correlated with the water discharge [Carey, 1985; Gomez, 1991; Bunte and Abt, 2005; Kuhnle and Willis, 1998; Ancey et al., 2006; Singh et al., 2009; Radice, 2009; Ganti et al., 2009]. As a result of sediment transport, alluvial river beds do not remain planar, but evolve toward a wavy morphology, which is continuously changing with time and gives rise to sediment waves that travel downstream or upstream depending on the Froude number [Julien, 1994; Coleman and Melville, 1996]. In most 
investigations of bed dynamics, bed forms are seen as the impact of turbulent structures on an erodible bed [Yalin, 1992] or the consequence of a loss of stability in the coupled fluid-solid system [Furbish et al., 1998; Balmforth and Provenzale, 2001]. More recently has emerged the idea that bed forms arise from the random nature of particle trajectories. For instance, using a discrete element model with simple rules for particle entrainment and motion, Niño et al. [2002] simulated individual particle trajectories and tracked the resulting bed evolution; they found that their model successfully mimicked many of the physical features of bed forms.

[4] These observations of the stochastic nature of bed load transport have given impetus to a probabilistic formulation of bed load transport equations. While Einstein [1936, 1950] extensively studied some stochastic features of bed load transport, in particular the probability distribution of heap lengths and travel times, he did not devise a complete probabilistic framework for sediment transport. Today there is renewed interest in working out an appropriate probabilistic framework, in particular driven by the need to overcome some limitations of the active layer concept that underpins most morphodynamic models. A first step toward this objective was achieved by Parker et al. [2000], who used heuristic arguments to derive a probabilistic formulation of the Exner equation in terms of bed-elevation-specific entrainment and deposition rates. Blom et al. [2008, 2006] and Blom and Parker [2004] elaborated on this model, with emphasis given to river beds covered by regular spatial patterns (triangular dunes). Jerolmack and Mohrig [2005] used a Langevin-like formulation of the Exner equation: they numerically solved a coupled set of equations, including the Exner equation where a white noise term was added to account for bed load fluctuations. They observed that adding noise in the Exner equation caused the formation of spatial patterns along the bed, which looked like natural bed forms in that they exhibited a continuous range of sizes and shapes (from ripples to dunes) and that they grew and moved in a similar way to what it is commonly observed in the field. Nakagawa and Tsujimoto [1980, 1984] showed that entrainment and deposition rates are in fact related to each other through the probability distribution of step lengths, which enables consideration of stochastic fluctuations in the Exner equation.

[5] Sediment transport is not the only physical system that is driven (to a varying degree) by fluctuations. Brownian motion, population dynamics, crystal growth, chemical reactions, landscape, earthquakes provide typical examples of stochastic systems [Turcotte, 1995; Sornette, 2000]. For these extensively studied problems, a longstanding issue has been to devise computational strategies to model complex systems by distinguishing deterministic and stochastic components of the behavior and applying appropriate generic procedures. There are some analogies that we can draw between sediment transport in waterways and systems made up of a large number of chemically reacting molecules: motion, entrainment, and deposition can be viewed as reactions between two species (moving and resting particles), as shown in Appendix B. In so doing, we can use all the mathematical machinery developed to build up a consistent theoretical framework and work out computational tools (Fokker-Plank equation, approximation by Ornstein-Uhlenbeck process, large-system limit expansion) [Gardiner, 1983]. A lesson learned in chemical kinetics is of particular relevance to our issue: in a well-stirred and large system (i.e., a system made up of a large number of molecules occupying all the available space), how the concentration of each chemical species evolves in time can be described using deterministic, first-order ordinary differential equations (called the reaction rate equations) [Gillespie, 2007]. This approximation breaks when molecules are present in small numbers, when reactions occur at low rates, or when molecules are heterogeneously distributed in the system; in that case, large fluctuations affect the system evolution to the point that dynamics are markedly different from the predictions of reaction rate equations.

[6] The objective of this paper is to recast the Exner equation in a probabilistic form (Langevin-like equation). Given the complexity of this issue, we will focus here on planar beds along which bed forms (nascent waves, ripples) start to develop. To that end, we will use an Eulerian approach and idealize particle entrainment and deposition as a birthdeath Markov process, which make it possible to track the time variation in the number of moving particles $N(t)$ inside a fixed control volume $\mathcal{V}$. The statistical properties of the time evolution of $N$ can be investigated using Markov process theory; this was presented at length in an earlier paper [Ancey et al., 2008]. Here, we are interested in determining how these properties change in the large-system limit (i.e., when the size of $\mathcal{V}$ is increased). The consequences in terms of sediment mass balance can then be inferred.

[7] To begin with, we outline the statistical model developed by Ancey et al. [2008] and compare it with other stochastic approaches, most of them being Lagrangian descriptions of particles experiencing random walks. A key issue is the definition of the solid discharge. As shown in Appendix A, there are many equations that express sediment flux, but there is no guarantee that they are all compatible and can equally serve within a probabilistic framework. We use a relation that links the solid discharge with the number of moving particles. A generalized birth-death Markov process is used to compute the number of moving particles $N(t)$ as well as the entrainment and deposition rates; see also Ancey et al. [2008] and Appendix B for details. In section 3.2, we will see how the streambed evolves under our model. We focus our attention to initially planar beds and low sediment transport rates (i.e., for bottom shear stresses slightly in excess of the threshold of incipient motion); as a result of sediment transport, bed forms start to appear in the form of ripples (i.e., bed forms with wave heights less than a few centimeters) [Coleman and Nikora, 2009]. Bed dynamics is accounted for through the Exner equation. When the system contains a sufficiently large number of particles, the master equation, which specifies how the probability of $N(t)$ evolves with time, can be conveniently transformed into a Fokker-Planck equation, which considers $N(t)$ as a continuous random variable rather than a discrete one. This transformation makes it possible to derive a probabilistic variant of the Exner equation in section 3.2. The discrete and continuous probability distributions of $N(t)$ are then compared with experiments. In the few experiments presented here, flow conditions are close to those encountered with shallow mountain streams and coarse sediment, but in principle our framework holds for a wide range of waterways provided that the physical assumptions used in the model (sediment flux is due to bed load transport 


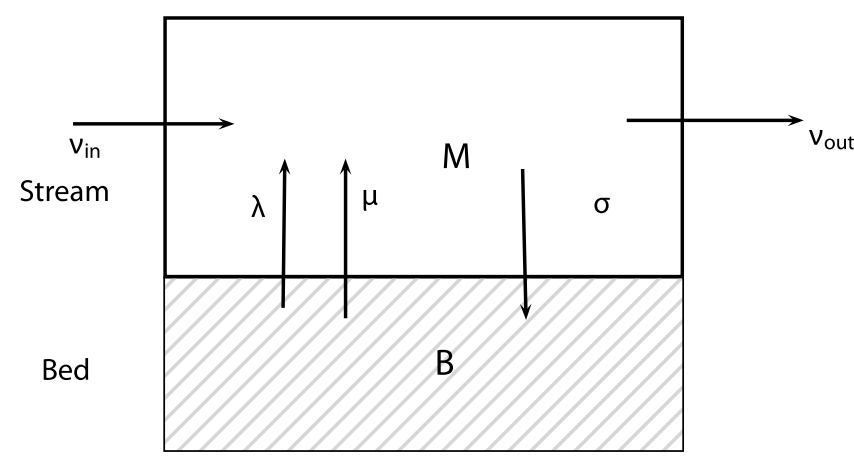

Figure 1. The number of moving particles observed within the window varies with time depending on the number of particles entering/leaving the window or being entrained/ deposited from/on the stationary bed.

and not suspended load) make sense. Except for some crude analytical approximations, there is no analytical solution to the stochastic differential equations that specify the system evolution. Numerical schemes must then be used (see section 3.6); I here provide proof of concept without dwelling on the algorithmic aspects.

[8] The notation used in this paper tries to be as much standard as possible. Greek letters generally denote parameters, e.g., entrainment and deposition coefficients in our model. Roman letters are used for random variables and the particular value they can take is denoted by lowercase letters. For instance, $N$ is a random variable describing the number of moving particles; the probability that $N$ takes the value $n$ is denoted by $P(n)=\operatorname{Pr}(N=n)$. The average or expectation of a distribution is denoted by a bar, e.g., $\bar{N}$ is the mean number of particles; for the sake of simplicity, I use the same symbol to refer to sample averages and theoretical expectations. The variance is denoted var. The symbol $o$ is a shorthand notation for 'much smaller than,' e.g., $u=o(v)$ means that $u \ll v$. The symbol $O$ indicates that there is a one-sided bound, e.g., $u=O(v)$ means that the limit of $u / v$ exists and is finite (neither zero nor infinity). Here probability functions $P$ are probability density functions (or probability mass functions for discrete variables). A variable index can be found at the end of this paper.

\section{Model Outline}

\subsection{Earlier Results}

[9] We have recently developed a model that computes the number of moving particles inside a control volume [Ancey et al., 2008]. The problem we addressed was an idealization of bed load transport in a mountain stream, where sediment was replaced by spherical particles of equal size and the problem was purely two dimensional. We adopted an Eulerian point of view by considering a fixed volume of control. Figure 1 depicts the control volume in which we count the number $N$ of particles. This number $N$ varies with time as a result of inflow, outflow, deposition, and entrainment of particles from the bed. More specifically, we assumed that particles enter and leave the window at rates $\nu_{\text {in }}$ and $\nu_{\text {out }}$ (in beads/s and $1 / \mathrm{s}$, respectively). In other words, the probability that one particle enters the window within a small time increment $\delta t$ is $\nu_{i n} \delta t$; the probability that two particles enter the window at the same time or over short time spans $\delta t$ is zero, which is a reasonable assumption for dilute to moderately concentrated bed load transport. For outflow, the probability that a given bead leaves the window is $\nu_{\text {out }} \delta t$, but since there are $N=n$ particles within the window, the probability that any particle leaves the window is $n \nu_{\text {out }} \delta$. For entrainment, we consider two processes: a particle can be dislodged from the bed as a result of fluid action (this is the classical assumption in sediment transport), but it can also be destabilized because of the moving particles, which can interact directly (collision) or indirectly (wake effect, advection of turbulent structure) with the bed particles. Individual entrainment occurs at rate $\lambda$, while collective entrainment is at rate $\mu$; entrainment rate is then dependent on the number of moving particles: $E \propto \lambda+\mu N$. For deposition, we could also distinguish various processes, but they are all dependent on $N$ and therefore, we just refer to $\sigma$ as the total deposition rate.

[10] Particle exchanges can be described using a birth-death emigration-immigration Markov model (see Appendix B). Under steady state conditions, several interesting features can be derived. First of all, when $\mu>0$ it can be shown that the probability of finding $N=n$ particles in the control volume is the negative binomial distribution

$P(n)=\operatorname{NegBin}(n ; r, p)=\frac{\Gamma(r+n)}{\Gamma(r) n !} p^{r}(1-p)^{n}, n=0,1, \ldots$,

with $r=\left(\lambda+\nu_{\text {in }}\right) / \mu$ and $p=1-\mu /\left(\sigma+\nu_{\text {out }}\right)$, and where $\Gamma$ denotes the gamma function. The mean and the variance are given by

$$
\begin{aligned}
\bar{N} & =r \frac{1-p}{p}=\left(\lambda+\nu_{\text {in }}\right) /\left(\sigma+\nu_{\text {out }}-\mu\right) \text { and } \\
\operatorname{var} N & =r \frac{1-p}{p^{2}}=\frac{\left(\lambda+\nu_{\text {in }}\right)\left(\sigma+\nu_{\text {out }}\right)}{\left(\sigma+\nu_{\text {out }}-\mu\right)^{2}},
\end{aligned}
$$

respectively. When $\mu=0, P(n)$ is the Poisson distribution and in that case, the variance equals the mean.

[11] Although the negative binomial distribution does not belong to the family of heavy-tail distributions (i.e., with an infinite variance), it has a tail falling off like $n^{r}(1-p)^{n}$. Its decay rate is thus slower than for an exponential distribution. Furthermore, when $p \rightarrow 0$, the coefficient of variation $\sqrt{\operatorname{var}} / \bar{N}$ tends to $\sqrt{r}$. This coefficient of variation can be much larger than unity, giving rise to large fluctuations and the appearance of a heavy-tail behavior. Note that this behavior is not shared by other classical discrete distributions such as the Poisson or binomial distribution. Continuous probability distributions used specifically in sediment transport for describing fluctuations such as Hamamori's distribution (5) are also characterized by a constant coefficient of variation (see section 2.2). Here, the potentially large variance results from what we referred to as collective entrainment. If we set $\mu$ to 0 , we retrieve an Einstein-like behavior, with a Poissonian distribution for $N$ [Ancey et al., 2006, 2008]. Note also that in our model, the jump probability depends on the number of moving particles (inside the control volume), which is affected by immigration and emigration, i.e., by its 
neighborhood. It thus describes a nonlocal process, where the local state depends on inflow and outflow.

[12] The second key feature is the autocorrelation function $R(t)$ of the number of particles in motion (within the control volume), which is exponential

$$
R(t)=e^{-t / t_{c}},
$$

with $t_{c}=1 /\left(\sigma+\nu_{\text {out }}-\mu\right)$ the autocorrelation time. When $\mu>0$, the autocorrelation time is quite long compared to the characteristic time of a moving particle $t_{m}=1 / \sigma$, which probably reflects a memory effect due to the coupling between entrainment and the number of moving particles. This also shows that even though birth-death Markov models are based on vanishing time increments $\delta t$ in the counting process, the typical time scale of $N$ is very large compared to particle time scale $\left(t_{m}\right.$ or $\left.\delta t\right)$.

[13] The third feature that can be derived under this model is the waiting time between events. Once in motion, a particle continues moving for a random, exponentially distributed time (with parameter $\sigma$ ); the time for which the particle is stationary has a far more complicated distribution, which cannot be specified in practice here. Indeed, there are major impediments to this determination: in our Eulerian approach, the details of a particle's history are ignored; furthermore, the waiting time for entrainment depends on the depth at which the particle was buried in the bed. Therefore, contrary to diffusion theory (see below), there is no clear relation between Lagrangian and Eulerian points of view within our framework. Other waiting times can be more easily computed. For instance, the waiting time between two deposition events within the control volume is distributed exponentially with a rate $1 /(n \sigma)$.

[14] There is no unique way to define the solid discharge and the statistical properties of the solid discharge depend greatly on the form selected. In Appendix A, we review the main forms that are currently of common use and comment on their strengths and weaknesses. Here we define the sediment transport rate $\dot{n}=Q_{s} / v_{p}$ as

$$
\dot{n}=\frac{1}{L} \sum_{i=1}^{N} u_{i}=\frac{N}{L} \bar{u}_{p}
$$

where $\bar{u}_{p}$ denotes the mean particle velocity, $v_{p}$ is the particle volume, and $Q_{s}$ is the total solid discharge. Fluctuations in the solid discharge arise from the variations in the number of moving particles in the control volume as well as their velocities. Surprisingly enough, we observe that the particle velocity $\bar{u}_{p}$ weakly contributes to the solid-discharge variance when the bed is mobile [Böhm et al., 2004; Ancey et al., 2008]; furthermore, contrary to the case in which a single particle is moving, collective motion of particles implies a weak dependence of particle velocity on bottom shear stress (see Appendix A). For a slope range of $7.5 \%-15 \%$ and a sufficiently wide range of bottom shear stress (above the threshold of incipient motion), Ancey et al. [2008] observed no dependence of the particle velocity for the rolling regime and weak dependence for the saltating regime; moreover the statistical properties of $N$ and $Q_{s}$ (e.g., the shape of the empirical probability distribution function and the autocorrelation function) were very close, confirming that $N$ is the key to understanding bed load fluctuations at low sediment transport rates. Using a wide flume, M. W. Schmeeckle (personal communication, 2007) obtained the same results concerning the weak dependence of $\bar{u}_{p}$ on $\tau_{b}$. These experimental observations prompt the use of $N$ as the main factor explaining the $Q_{s}$ variance. More complicated dependences can be accounted for without significantly altering the structure of the model here. For instance, Böhm et al. [2004] showed how to combine both $N$ and $\bar{u}_{p}$ fluctuations to compute the statistical properties of $\dot{n}$.

\subsection{Comparison With Other Approaches}

[15] There are numerous approaches to computing the solid discharge, but only a few yield both the dependence on water flow rate and its statistical properties. Einstein [1936, 1950] devised a simple model where bed load transport results from the imbalance between entrainment and deposition rates. Fluctuations arise from the intermittent motion of particles: once moving, particles perform steps of random length and once they are deposited, the periods of rest are also of random span. Note that setting $\mu=0$ (i.e., no collective entrainment) in our model leads to an Einstein-like model. This model has seen many refinements, the most popular being Hamamori's distribution which gives the probability density function of the scaled variables $z=\left(Q_{s}-\bar{Q}_{s}\right) / \sqrt{\operatorname{var} Q_{s}}$

$$
P(z)=-\frac{\sqrt{7}}{12} \ln \left(\frac{\sqrt{7}}{12} z+\frac{1}{4}\right)
$$

where $\bar{Q}_{s}$ is the averaged solid discharge and the variance is related to this mean value by: $\operatorname{var} Q_{s}=7 \bar{Q}_{s}^{2} / 9$, that is, a coefficient of variation of $\sqrt{7} / 3$ [Carey, 1985; Kuhnle and Willis, 1998]. Note that this distribution is bounded since $-3 / \sqrt{7}<z<9 / \sqrt{7}$, which imposes that the maximum amplitude of fluctuations (around the mean value) is four times the mean rate.

[16] In recent years, growing attention has been paid to models based on diffusion theory. Originally, linear diffusion theory was used to model erosion processes in sedimentary basins over geological timescales [e.g., see Paola et al., 1992]. In the particular case of a one-dimensional stream, the governing equation takes the form of a parabolic partial differential equation

$$
\frac{\partial b(x, t)}{\partial t}=\frac{\partial}{\partial x}\left(K \frac{\partial b(x, t)}{\partial x}\right)
$$

with $b(x, t)$ the bed elevation and $K$ is a constant reflecting soil diffusivity. Field surveys giving little hope for linearity [Heimsath et al., 2005], emphasis was then given to nonlinear forms of (6), where $K$ is assumed to be a function of $b$ [Postma et al., 2008], and anomalous diffusion, where derivatives are replaced with fractional derivatives [Voller and Paola, 2010; Stark et al., 2009].

[17] It is also very tempting to use advection-diffusion equations to model sediment transport on shorter time scales than geological times [Graf, 1984]. For instance, the fol- 
lowing equation describes how the concentration in particles varies with time and position

$$
\frac{\partial c}{\partial t}=-u \frac{\partial c}{\partial x}+\frac{\partial}{\partial x}\left(K \frac{\partial c}{\partial x}\right)
$$

where $u$ is the mean velocity at which the particles are transported and $K$ is a (constant) diffusion coefficient. A particular strength of this formulation is that it can be derived using an Eulerian continuum-based approach or a Lagrangian discrete particle approach. The correspondence between both approaches is detailed at length by Schumer et al. [2009], but it is worth recalling some fundamentals here. The solid concentration is the volume fraction occupied by particles and is related to the probability $P_{1}(x, t)$ of finding a particle placed at $x$ at time $t$ [Herczynski and Pienkowska, 1980]:

$$
c(x, t)=N P_{1}(x, t)
$$

with $N$ the number of particles. Solving (7) for $c$ is then equivalent to finding the probability $P_{1}(x, t)$, which gives an unusually simple relation for connecting probabilistic and deterministic descriptions. If the flow of particles is dilute, we can reasonably assume that the motion of a single particle can be closely described using a random walk: the particle randomly jumps from one position to another one. The jumps of length $X_{i}$ are randomly distributed with mean $m$ and finite variance $v$. There is no waiting time between jumps. The times of flight are constant and equal to $\Delta t$. The distance $d$ traveled by the particle at time $t$ is

$$
d(t)=\sum_{i=1}^{k} X_{i}
$$

where $k$ is the number of jumps achieved until time $t$; for sufficiently large values of $t$, we have $k \approx t / \Delta t$. The law of large numbers states that the sum of independent and identically distributed random variables (with finite variance $v$ ) scaled by $\sqrt{v k}$ converges to the standard normal distribution $\mathcal{N}(0,1)$ (of mean 0 and variance 1 )

$$
\frac{\sum_{i=1}^{k} X_{i}-k m}{\sqrt{v k}} \rightarrow \mathcal{N}(0,1)
$$

which can be recast in the following form

$$
d(t) \approx \frac{m}{\Delta t} t+\sqrt{\frac{v}{\Delta t}} \sqrt{t} \mathcal{N}(0,1) .
$$

This shows that the probability $P_{1}(x, t)$ of finding the particle at $x=d$ is given by a normal law with mean $m / \Delta t$ and variance $v / \Delta t$. The random walk model can be made more complex, e.g., by considering infinite variance for particle jumps or by allowing random waiting times between jumps. This results in fractional advection-diffusion equations, widely used to investigate solute transport in groundwater [Berkowitz et al., 2006]. A key feature is that the probability distribution $P_{1}$ (or the solid concentration) is a heavy-tailed distribution, which explains why wide and frequent fluctuations occur in solute transport. For instance, if the distri- bution of particle step lengths is heavy tailed (i.e., with infinite variance), then $P_{1}$ or $c$ satisfies a fractional-in-space advection-diffusion equation, which generalizes the classic advection-diffusion equation [Metzler and Klafter, 2004; Schumer et al., 2009]:

$$
\frac{\partial c}{\partial t}=u \frac{\partial c}{\partial x}+K \frac{\partial^{\gamma} c}{\partial x^{\gamma}}
$$

where $0<\gamma<2$ is the fractional exponent and we introduced the fractional derivative

$$
\frac{\partial^{\gamma} c}{\partial x^{\gamma}}=\frac{1}{\Gamma(1-\gamma)} \int_{0}^{x} \frac{\partial c(x-y, t)}{\partial x} y^{-\beta} d y
$$

The solution to (12) has no analytical expression, but one can infer its main features: it is positively skewed and has a tail that falls off like $x^{-\gamma-1}$, i.e., much slower than the tails of exponential or Gaussian distributions. Note that since the fractional derivative (13) is equivalent to a convolution with a power law weighting function, fractional diffusion is a nonlocal process [Tucker and Bradley, 2010].

[18] The question arises as to whether the fractional diffusion framework will help improve our understanding of bed load transport. Although there is abundant literature in porous medium and groundwater problems on fractional diffusion equations and random walks, there are not many results available for sediment transport. Much of the literature has focused on tracers in gravel bed rivers and flumes. By observing the final distribution of particle displacements, Einstein [1936] and Hubbell and Sayre [1964] deduced that the deposition patterns are consistent with a gamma distribution of step lengths. On the whole, subsequent field surveys led to similar conclusions, but they also pointed out that data were noisy and the final distribution of particle displacements could be complicated since particles could be trapped in river bars and their movement could be affected by local hydraulic conditions depending on channel morphology [Hassan and Church, 1991; Schmidt and Ergenziger, 1992; Pryce and Ashmore, 2003; Ferguson et al., 2002; McNamara and Borden, 2004]. More recently, new techniques such as radio tracking has made it possible to follow up the travel path of individual particles; Habersack [2001] reported that for observation time scales of a few minutes, the rest periods are exponentially distributed, while the distribution of step lengths is closely approximated by a gamma distribution. In contrast with other authors, Nikora et al. [2002] focused on particle movement as a function of time rather than step length distribution at a given time. From their flume experiments, they deduced that $\operatorname{var} d(t) \propto t^{\gamma}$ with $\gamma \geq 2$ for short time scales (typically on the order of a few seconds), but $\gamma<2$ for long time scales (typically on the order of a few minutes), which means that particle displacements exhibit anomalous diffusive properties, either superdiffusive or subdiffusive depending on the time scale. This behavior can be understood by considering that the longer the time scale is, the more likely the particle is to be trapped in the bed. In summary, while there is growing evidence of wide and frequent fluctuations in bed load transport (in particular at low flow rates) [Carey, 1985; Kuhnle and Southard, 1988; Kuhnle and Willis, 1998; Bunte and Abt, 2005; Ancey et al., 2006; Singh et al., 2009; Radice, 2009], the origins of these fluctuations are 


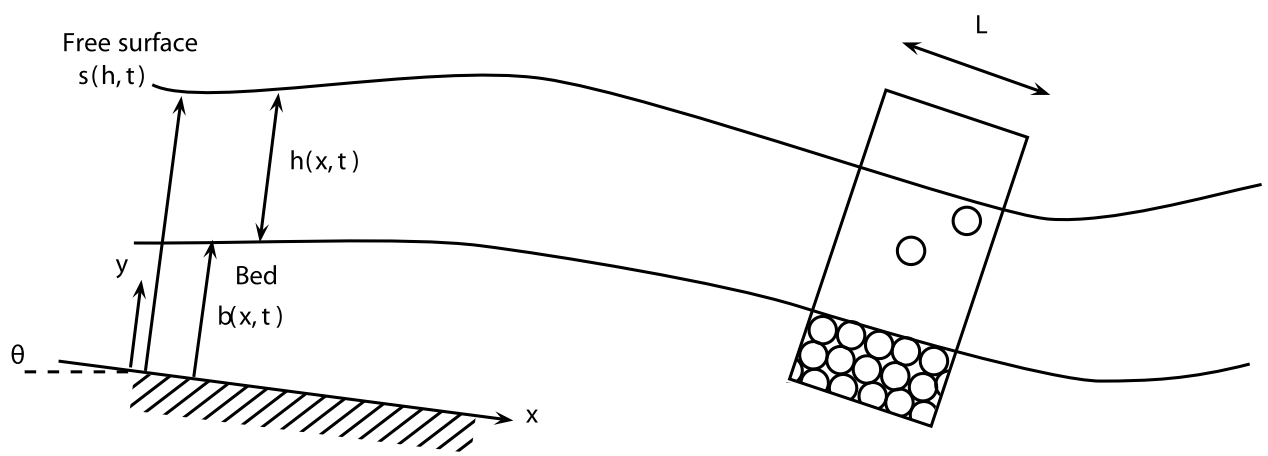

Figure 2. Definition sketch of the model. The reference level is the $x$ axis.

not clear-cut: while there are some data supporting the idea of fractional diffusion [Nikora et al., 2002], the few data collected so far in the field or laboratory do not reveal typical fractional diffusion behavior for particle displacements or bed load concentration.

\section{Bed Dynamics and Exner Equation}

\subsection{Setting}

[19] We consider a two-dimensional, steady water stream flowing down an erodible bed $y=b(x, t)$, with $x$ the downstream spatial coordinate, $t$ time, and $y$ the vertical coordinate measured from a fixed reference level. The $x$ axis is tilted at a constant angle $\theta$ to the horizontal. Moving and resting particles are spherical particles of equal radius $a$ and density $\varrho_{p}$. The free surface is at elevation $y=s(x, t)$ and the flow depth $h(x, t)=s(x, t)-b(x, t)$ is measured normal to the reference level (i.e., in the $y$ direction). Figure 2 shows the flow configuration.

\subsection{Exner Equation and Bed Dynamics}

[20] Even under bed equilibrium conditions, the bed does not remain flat and bed forms develop. We must then use an equation specifying the bed mass balance. When the suspended load stored in the water column is negligible compared to bed load, the Exner equation fulfills this objective

$$
\left(1-\phi_{p}\right) \frac{\partial b}{\partial t}=D-E=-\frac{\partial q_{s}}{\partial x}
$$

where $\phi_{p}$ is the bed porosity, $D(E)$ denotes the volume rate of deposition (entrainment) per unit time and per unit bed surface onto the bed, and $q_{s}$ represents the solid discharge per unit width.

[21] The entrainment and deposition rates are assumed to be single-valued functions of the bottom shear stress: $D=$ $D\left(\tau_{b}\right)$ and $E=E\left(\tau_{b}\right)$. Under steady uniform flow conditions, the bottom shear stress is $\tau_{b}(h)=\rho g h \sin \theta$. Here we focus on planar bed lower-regime flow conditions, which describe situations where the bed remains nearly planar even though bed forms start to appear as a result of incipient bed load transport [Julien, 1994; Coleman and Nikora, 2009]. The water stream is in a nearly uniform or gradually varied regime and is not yet disturbed by nascent bed forms. Sediment transport occurs at low rate; that is, the bottom shear stress $\tau_{b}$ just exceeds the threshold of incipient motion $\tau_{c}$ or in other words, the transport stage parameter $T$ is low, typically $T<3$ with $T=\tau_{b} / \tau_{c}-1$ [van Rijn, 1984]. In this case, the bottom shear stress remains, as a first approximation, very close to its value in a steady uniform regime. A first-order correction of the uniform regime is

$$
\tau_{b}(\bar{u}, b, h)=\rho g h \cos \theta\left(\tan \theta-\frac{\partial b}{\partial x}-\frac{\partial h}{\partial x}\right)
$$

which accommodates for both slight changes in bed gradient $\partial_{x} b$ and flow-depth deviations from the uniform regime $\partial_{x} h$ [Julien, 1994].

[22] Under our model, the entrainment and deposition rates are found to depend on the number of moving particles $N$ as follows:

$$
E=\frac{v_{p}}{L}(\lambda+\mu N), \text { and } D=\frac{v_{p}}{L} \sigma N,
$$

where $\lambda$ (in beads/s), $\mu$ (in $\mathrm{s}^{-1}$ ), and $\sigma$ (in $\mathrm{s}^{-1}$ ) are functions of $\tau_{b}, v_{p}=4 \pi^{3} / 3$ denotes the particle volume and $L$ is the length of the control volume. The entrainment rate $\lambda$ is also proportional to $L$, while the exchange rates $\sigma$ and $\mu$ are independent of it. We can then introduce an entrainment rate per unit length $\left(\tilde{\lambda}\right.$ in beads $\left.\mathrm{m}^{-1} \mathrm{~s}^{-1}\right)$, a scaled outflow rate ( $\tilde{\nu}_{\text {out }}$ in $\mathrm{m}^{-1} \mathrm{~s}^{-1}$ ), and the concentration of moving particles per unit length $\left(\tilde{N}\right.$ in beads $\left.\mathrm{m}^{-1}\right)$ :

$$
\lambda=L \tilde{\lambda}, \nu_{\text {out }}=\frac{\tilde{\nu}_{\text {out }}}{L}, \text { and } N=L \tilde{N} .
$$

This scaling holds since (1) for entrainment, the dependence on $L$ is encoded through $N$ for collective entrainment $(\mu)$, while for individual entrainment, the longer the window is, the more probable particle entrainment is, and (2) for outflow, as illustrated by Figure 5 in section 3.4 or more rigorously by computing intercorrelation times [Böhm et al., 2004], the outflow rate is related to the mean particle velocity (i.e., $\tilde{\nu}_{\text {out }} \approx u_{p}$ ). We can then express the entrainment and deposition rates as follows:

$$
E=\frac{v_{p}}{L}(\lambda+\mu N)=v_{p}(\tilde{\lambda}+\mu \tilde{N}) \text { and } D=\frac{v_{p}}{L} \sigma N=v_{p} \sigma \tilde{N}
$$

The Exner equation (14) becomes

$$
\psi \frac{\partial b}{\partial t}=(\sigma-\mu) \tilde{N}-\tilde{\lambda}
$$


with $\psi=1-\phi_{p}$. Equation (19) is a stochastic differential equation because $\tilde{N}$ is a random variable, the variations of which are described by the master equation (20) or the approximate governing equation (27), as shown in section 3.3. This makes the analysis and numerical treatment of (19) somewhat delicate and prompts us to seek analytical approximation of $N$.

\subsection{Behavior of $N$ in the Large-System Limit}

[23] The model outlined in section 2.1 is a birth-death Markov process, which allows jumps only one step up or down [Cox and Miller, 1965] over short time spans. For $n \geq 1$, the governing equation that specifies the probability of finding $N=n$ particles in the control volume is

$$
\begin{aligned}
\frac{\partial}{\partial t} P(n ; t)= & (n+1) \alpha P(n+1 ; t)+\{\beta+(n-1) \mu\} P(n-1 ; t) \\
& -\{\beta+n(\alpha+\mu)\} P(n ; t)
\end{aligned}
$$

with the shorthand notation $\alpha=\sigma+\nu_{\text {out }}$ and $\beta=\lambda+\nu_{\text {in }}$. For $n=0$, we have

$$
\frac{\partial P(0, t)}{\partial t}=\alpha P(1 ; t)-\beta P(0 ; t)
$$

At time $t=0$, there are $N=N_{0}$ particles within the control volume, so we set

$$
P(n ; 0)=\delta\left(n-N_{0}\right)
$$

where $\delta$ is the Kronecker delta function. The forward master equation (20) offers some resistance to analysis. There are some general techniques based on an appropriate transformation of the dependent variable (e.g., Laplace transform and generating functions) to find analytical solutions to the differential difference equation (20), but the inverse transformation is not always easy to handle; for instance Ancey et al. [2008] provided a general solution to (20) in terms of the generating function $G(z, t)=\sum_{n=0}^{\infty} z^{n} P(n ; t)$. For steady flow conditions, it can be shown that the solution to (20) is a negative binomial distribution (1).

[24] Here we proceed differently by seeking an analytical approximation of (20) for sufficiently large $N$ values. The idea is to transform (20) into a Fokker-Plank equation, which provides further insight into the stochastic behavior of bed load transport. There are standard techniques (KramersMoyal and van Kampen expansions) described in the technical literature to achieve such a transformation [Gardiner, 1983]. Using a less rigorous, but far easier and more intuitive approach, we will proceed differently: essentially, we interpret the contributions $P(n+1)$ and $P(n-1)$ as the terms arising in a Taylor series expansion of $P(n)$ since to first order and $n$ sufficiently large (compared to unity), we have $P(n+1)-P(n-1)=4 P^{\prime}(n)$ and $P(n+1)-2 P(n)+P(n-1)=$ $P^{\prime \prime}(n)$. In the large-number limit, we find that the forward master equation (20) can be approximated by

$$
\frac{\partial}{\partial t} P(n ; t)=-\frac{\partial}{\partial n}(A P)+\frac{1}{2} \frac{\partial^{2}}{\partial n^{2}}(B P),
$$

where $A=\beta+n(\mu-\alpha)$ is called the drift function and $B=$ $(\alpha+\mu) n+\beta$ is the diffusion function. Function $A$ describes the deterministic component, while function $B$ reflects the random fluctuations of sediment transport rate. Indeed when $B$ is zero, (23) is strictly equivalent to the nonlinear deterministic (Liouville) differential equation $d N / d t=A(N)$ (see (29) thereafter); when $A$ is zero and $B$ is constant, (23) describes a special Wiener process (i.e., the random walk of a Brownian particle). A net advantage of (23) compared to (20) is that we better understand how the different exchanges between the bed and the stream gives rise to effects that can be interpreted as deterministic process and stochastic diffusion at the macroscopic scale (see also Gillespie [1992, chapter 6] for further discussion).

[25] Another equivalent and useful form of the FokkerPlanck equation (23) is the (Ito) stochastic differential equation [Gardiner, 1983]:

$$
d N=A(N) d t+B^{1 / 2}(N) d \mathcal{W}(t)
$$

where $\mathcal{W}(t)$ is the Wiener process. In the following, we will use this equation in the Exner equation to evaluate the effect of $N$, but prior to this, we will make a change of variables that transforms the discrete random variable $N$ into a continuous one $\tilde{N}$. We can express

$$
\begin{gathered}
A=L\left(\tilde{\lambda}+\frac{\nu_{\text {in }}-\tilde{N} \tilde{\nu}_{\text {out }}}{L}+\tilde{N}(\mu-\sigma)\right) \text { and } \\
B=L\left((\sigma+\mu) \tilde{N}+\tilde{\lambda}+\frac{\nu_{\text {in }}+\tilde{N} \tilde{\nu}_{\text {out }}}{L}\right),
\end{gathered}
$$

which shows that in the large-system limit $(L \rightarrow \infty)$, the following approximations hold to first order

$$
\begin{gathered}
\tilde{A}=\frac{A}{L}=\tilde{\lambda}+\tilde{N}(\mu-\sigma)+O(1 / L) \text { and } \\
\tilde{B}=\frac{B}{L}=\tilde{\lambda}+(\sigma+\mu) \tilde{N}+O(1 / L) .
\end{gathered}
$$

This change of variables turns out very helpful in that the system size appears in the governing equations, which is crucial to simplifying these equations. Equation (24) can now be expressed as a function of $\tilde{N}$ :

$$
d \tilde{N}=\tilde{A}(\tilde{N}) d t+\frac{1}{\sqrt{L}} \tilde{B}^{1 / 2}(\tilde{N}) d \mathcal{W}(t) .
$$

In the large-system limit, the factor $\epsilon=L^{-1 / 2}$ tends toward zero, implying that the stochastic fluctuations generating weak noise in the time evolution of $\tilde{N}$ cancel out; in other words, the system is entirely driven by the deterministic component $\tilde{A}$. This leads to posing a power series expansion of $\tilde{N}$

$$
\tilde{N}=\tilde{N}_{*}+\epsilon \eta_{1}(t)+\cdots .
$$

The zero-order term in this expansion is also called the sure function; it is the solution to the Liouville equation

$$
\frac{d \tilde{N}}{d t}=\tilde{A}(N)
$$




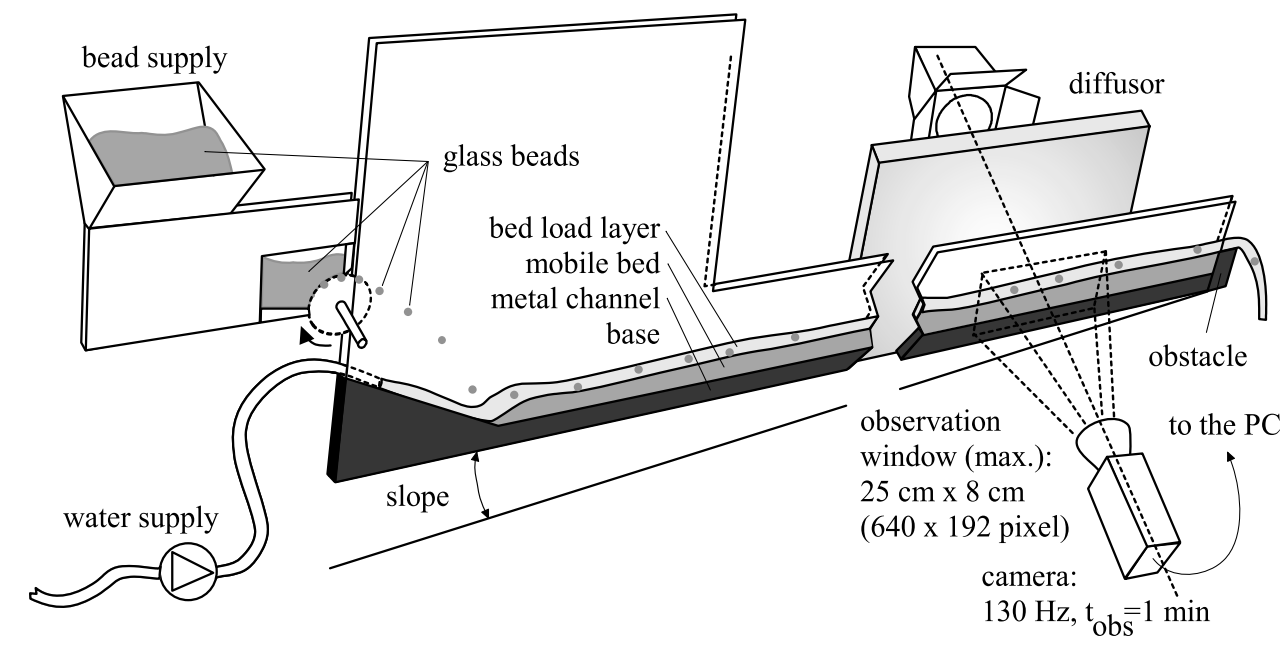

Figure 3. Sketch of the experimental setup.

subject to an initial condition in the form $\tilde{N}=\tilde{N}_{0}$ at $t=0$. When the bottom shear stress is constant or does not deviate significantly from its mean value, the solution is

$$
\tilde{N}_{*}(t)=\frac{\tilde{\lambda}-e^{-t(\sigma-\mu)}\left(\tilde{\lambda}+N_{0}(\mu-\sigma)\right)}{\sigma-\mu} .
$$

The number of moving particles decreases exponentially toward a constant value. The solution $N_{*}(t)$ represents the deterministic component of the behavior when the stochastic fluctuations are ignored. Note that at long times and for constant bottom shear stress, the number of moving particles approaches a stationary value

$$
\tilde{N}_{*}(t) \rightarrow \tilde{N}_{\infty}=\frac{\tilde{\lambda}}{\sigma-\mu},
$$

which is the steady state mean number [Ancey et al., 2008]. For large systems, the relaxation time is $t_{r}=(\sigma-\mu)^{-1}$.

[26] The first-order correction term $\eta_{1}$ is the solution to

$$
d \eta=(\mu-\sigma) \eta(t) d t+\tilde{B}^{1 / 2}\left(N_{*}(t)\right) d \mathcal{W}(t),
$$

subject to an initial condition in the form $\eta=0$ at $t=0$. Note that the diffusion function is now $B^{1 / 2}\left(N_{*}(t)\right)$, which implies that the fluctuation amplitude is imposed by the sure function $N_{*}$. From (32), we deduce that $\eta_{1}$ is a continuous Markov process with drift function $(\mu-\sigma) \eta_{1}(t)$ and diffusion function $\tilde{B}\left(N_{*}(t)\right)$; it represents additive noise. Since the drift function is linear and the diffusion function is constant when $t \rightarrow \infty$, we deduce that the long-time behavior of $\eta_{1}$ can be approximated by an Ornstein-Uhlenbeck process with parameter $k_{o}=\sigma-\mu$ and diffusion $D_{o}=\tilde{B}\left(\tilde{N}_{\infty}\right)$ [see Gardiner, 1983, chapter 3]. Since the long-time behavior of a Ornstein-Uhlenbeck process is a Gaussian process $\mathcal{N}$ with mean 0 and variance $D_{o} /\left(2 k_{o}\right)$, we deduce that

$$
t \rightarrow \infty, \eta_{1} \sim \mathcal{N}\left(0, \frac{D_{o}}{2 k_{o}}\right)=\mathcal{N}\left(0, \frac{\tilde{\lambda} \sigma}{(\sigma-\mu)^{2}}\right) .
$$

In other words, this also means that in the limit of $t \rightarrow \infty$, the number of moving particles follows a Gaussian distribution

$$
\tilde{N} \sim \mathcal{N}\left(\tilde{N}_{\infty}, \epsilon^{2} \frac{\tilde{\lambda} \sigma}{(\sigma-\mu)^{2}}\right),
$$

or equivalently $n \sim \mathcal{N}\left(N_{\infty}, \frac{\lambda \sigma}{(\sigma-\mu)^{2}}\right)$. The standard deviation of the particle concentration $\tilde{N}$ goes to zero with increasing $L$ like $L^{-1 / 2}$; this is the usual dependence on system size for thermodynamic systems [Gardiner, 1983].

\subsection{Experimental Validation}

[27] Experiments were performed in a tilted, narrow, glasssided channel, $2 \mathrm{~m}$ in length and $20 \mathrm{~cm}$ in height, as depicted in Figure 3. The channel width $W$ was adjusted to $6.5 \mathrm{~mm}$, which was slightly larger than the particle diameter $2 a=$ $6 \mathrm{~mm}$. In this way, particle motion was approximately two dimensional. The channel slope $\tan \theta$ ranged from $7.5 \%$ to $15 \%$.

[28] The channel base consisted of half-cylinders of equal size (radius $r=a$ ) and randomly arranged on different levels. Colored spherical glass beads with a density $\rho_{p}=2500 \mathrm{~kg} / \mathrm{m}^{3}$ (provided by Sigmund Lindner $\mathrm{GmbH}$, Germany) were injected from a reservoir into the channel using a wheel. For the experiments presented here, the injection rate $\dot{n}_{0}$ was 5-21 beads per second, with an uncertainty of less than 5\%. This corresponded to a solid discharge per unit width $q_{s}=$ $Q_{s} / W$ of $9-38 \times 10^{-5} \mathrm{~m}^{2} / \mathrm{s}$.

[29] The experimental conditions (velocity profile, bed friction, etc.) were specified in earlier papers [Ancey et al., 2008]. Although the flume was narrow, we checked that its hydraulic characteristics were like those observed in wide channels with shallow flows [Frey and Reboud, 2001]. The flow Reynolds number was in the 3000-7700 range. The Froude number varied significantly over the experimental duration and along the mainstream direction. Its mean values were close to 1.5 (in the $1-4$ range), which means that the flows were supercritical on average, but its instantaneous values fluctuated a great deal and frequent transitions to subcritical regimes occurred. Note that we defined the solid discharge $\dot{n}$ as the flux of beads per unit time. We can also 
Table 1. Flow Characteristics and Measured Values

\begin{tabular}{|c|c|c|c|c|c|c|c|c|c|c|c|c|c|c|c|}
\hline & \multicolumn{15}{|c|}{ Experiment Number ${ }^{\mathrm{a}}$} \\
\hline & E7-6 & E7-8 & E7-9 & E7-11 & E10-6 & E10-7 & E10-8 & E10-9 & E10-16 & E10-21 & E12-9 & E12-16 & E12-21 & E15-16 & E15-21 \\
\hline $\tan \theta(\%)$ & 7.5 & 7.5 & 7.5 & 7.5 & 10.0 & 10.0 & 10.0 & 10.0 & 10.0 & 10.0 & 12.5 & 12.5 & 12.5 & 15.0 & 15.0 \\
\hline$q_{w}\left(10^{-3} \mathrm{~m}^{2} / \mathrm{s}\right)$ & 10.00 & 11.54 & 13.85 & 26.15 & 4.15 & 4.42 & 5.38 & 5.54 & 8.19 & 10.31 & 2.97 & 3.85 & 4.46 & 2.31 & 2.92 \\
\hline$h(\mathrm{~mm})$ & 18.9 & 20.8 & 24.9 & 40.8 & 10.2 & 10.8 & 12.2 & 12.5 & 16.9 & 19.4 & 7.0 & 8.2 & 9.4 & 4.9 & 6.7 \\
\hline$u_{f}(\mathrm{~m} / \mathrm{s})$ & 0.53 & 0.55 & 0.56 & 0.64 & 0.41 & 0.41 & 0.44 & 0.44 & 0.48 & 0.53 & 0.42 & 0.47 & 0.48 & 0.47 & 0.44 \\
\hline$\dot{n}$ (beads/s) & 5.45 & 7.76 & 9.20 & 10.99 & 5.72 & 6.85 & 7.74 & 9.41 & 15.56 & 20.57 & 9.52 & 15.52 & 19.86 & 15.45 & 20.55 \\
\hline $\bar{u}_{p}$ & 0.16 & 0.17 & 0.15 & 0.15 & 0.11 & 0.13 & 0.11 & 0.12 & 0.13 & 0.12 & 0.08 & 0.09 & 0.10 & 0.07 & 0.08 \\
\hline$N_{r}$ & 4.62 & 6.01 & 8.33 & 9.96 & 8.31 & 7.51 & 10.42 & 11.35 & 18.15 & 26.55 & 20.00 & 30.15 & 33.73 & 41.87 & 45.56 \\
\hline$N_{s}$ & 2.25 & 3.22 & 3.94 & 5.21 & 2.35 & 2.99 & 3.28 & 3.85 & 6.09 & 7.74 & 1.83 & 3.09 & 4.86 & 0.84 & 3.42 \\
\hline $\operatorname{var} N$ & 22.53 & 30.94 & 35.43 & 38.51 & 70.74 & 36.89 & 64.55 & 49.09 & 72.09 & 129.91 & 123.38 & 182.68 & 222.88 & 214.31 & \\
\hline$\sigma(1 / \mathrm{s})$ & 4.67 & 5.28 & 5.04 & 4.90 & 5.12 & 4.86 & 4.94 & 4.73 & 4.52 & 4.39 & 4.83 & 4.77 & 4.51 & 4.20 & 3.90 \\
\hline$\mu(1 / \mathrm{s})$ & 3.73 & 4.14 & 3.80 & 3.46 & 4.96 & 4.07 & 4.33 & 3.91 & 3.55 & 3.66 & 4.33 & 4.32 & 4.15 & 3.64 & 2.73 \\
\hline$\lambda$ (beads/s) & 6.59 & 11.21 & 15.13 & 21.40 & 1.53 & 7.79 & 8.42 & 11.23 & 22.07 & 24.77 & 10.95 & 14.64 & 13.62 & 24.49 & 55.71 \\
\hline$\nu_{i n}($ beads $/ \mathrm{s})$ & 5.44 & 7.76 & 9.19 & 10.98 & 5.71 & 6.84 & 7.73 & 9.41 & 15.55 & 20.56 & 9.51 & 15.51 & 19.85 & 15.45 & 20.54 \\
\hline$\nu_{\text {out }}(1 / \mathrm{s})$ & 0.77 & 0.78 & 0.75 & 0.73 & 0.60 & 0.69 & 0.56 & 0.68 & 0.68 & 0.60 & 0.43 & 0.47 & 0.51 & 0.35 & 0.42 \\
\hline
\end{tabular}

${ }^{\mathrm{a}}$ E7-6, experiment a; E7-8, experiment b; E7-9, experiment c; E7-11, experiment d; E10-6, experiment e; E10-7, experiment f; E10-8, experiment g; E10-9, experiment h; E10-16, experiment i; E10-21, experiment j; E12-9, experiment k; E12-16, experiment 1; E12-21, experiment m; E15-16, experiment $\mathrm{n}$; E15-21, experiment 0.

define the solid discharge $Q_{s}$ as the volume flow rate and relate it $\dot{n}$ using $Q_{s}=\pi(2 a)^{3} \dot{n} / 6$. For $\dot{n}$, we used the same definition (4) as that used in the theoretical analysis. The bottom shear stress is evaluated as $\tau_{b}=\rho g h \sin \theta$ [Frey and Reboud, 2001].

[30] We ran 15 experiments (referred to as a-o) with different inclinations and various flow rates. Bed load equilibrium flows were achieved. The features of each run are summarized in Table 1 . We report the water discharge $q_{w}$, the mean flow depth $h$, the mean water velocity $u_{f}=q_{w} / h$, the solid discharge $\dot{n}$. The notation E10-6 means: $\tan \theta=$ $10 \%$ and $\dot{n} \approx 6$ beads/s.

[31] The particles and the water stream were filmed using a Pulnix partial scan video camera (progressive scan TM-6705AN), placed perpendicular to the glass panes at $115 \mathrm{~cm}$ away from the channel, approximately $80 \mathrm{~cm}$ upstream from the channel outlet. The camera was inclined at the same angle as the channel, behind which lights were positioned. An area of $L=22.5 \mathrm{~cm}$ in length and $8 \mathrm{~cm}$ in height was filmed. The camera resolution was $640 \times 192$ pixels for a frame rate of 129.2 fps (exposure time: $0.2 \mathrm{~ms}, 256$ gray levels). Each sequence comprised 8000 images because of limited computer memory; this corresponded to a duration of approximately one minute. Positions of the bead mass centers were detected by means of an algorithm combining several image processing operations; particle trajectories were calculated using a tracking algorithm [Böhm et al., 2006].

[32] Table 1 reports the mean particle velocity in the rolling regime $\bar{u}_{r}$, the mean particle velocity $\bar{u}_{p}$, the number of particles in a rolling regime $N_{r}$ and in a saltating regime $N_{s}$ (the total number of moving particles being $N=N_{r}+N_{s}$ ), the entrainment and deposition rates $\lambda, \sigma, \mu$ together with the inflow and outflow rates $\nu_{\text {in }}$ and $\nu_{\text {out }}$. The entrainment and deposition rates $\mu$ and $\lambda$ were estimated using (2) and the equilibrium conditions for the bed $\left(\sigma+\nu_{\text {out }}\right) \bar{N}=\nu_{\text {in }}+\lambda+\mu \bar{N}$ and the control volume $\nu_{\text {out }} \bar{N}=\nu_{\text {in }}$, where $\bar{N}$ is the mean number of moving particles. The deposition rate $\sigma$ was measured using image processing, while $\nu_{\text {in }}$ is imposed at the flume inlet.

[33] A striking result is the weak dependence of the mean particle velocity on bottom shear stress $\tau_{b}$. As shown in Figure 4, for a given slope, the mean particle velocity reaches a nearly constant level independently of $\tau_{b}$ (but depending on $\theta$ ). For instance, for $\theta=10 \%$, for $\tau_{b} \approx 10 \mathrm{~Pa}$ (run E10-6 in Table 1), the mean particle velocity is $\bar{u}_{p}=11 \mathrm{~cm} / \mathrm{s}$ against $\bar{u}_{p}=12 \mathrm{~cm} / \mathrm{s}$ for $\tau_{b} \approx 20 \mathrm{~Pa}$ (run E10-21 in Table 1). Using a wide flume, M. W. Schmeeckle (personal communication, 2007) observed the same behavior, which leads us to think that this behavior may be quite general for intermittent bed load transport conditions. Note that this behavior was induced by the experimental setup (in particular, the narrow flume), but a consequence of collective motion; when a single particle was in motion in the window, the particle velocity markedly depend on fluid velocity [Ancey et al., 2008].

[34] Interestingly enough, when we reported the emigration rate $\nu_{\text {out }}$ as a function of the particle velocity, we found out that $\nu_{\text {out }}=\bar{u}_{p} / L^{\prime}$, with $L^{\prime}=20 \mathrm{~cm}$, as shown in Figure 5 . This shows that the outflow rate is directly proportional to the mean particle velocity, which is to be expected. Indeed recall that theoretically, the particle flow rate was defined as the volume average of particle velocities $\dot{n}=N \bar{u}_{p} / L$ (see equation (4)) and that in a steady state, the flux of outgoing particles is $\nu_{\text {out }} N=\dot{n}$, which gives $\nu_{\text {out }}=\bar{u}_{p} / L$ under steady state conditions. Experimentally we found that this relation holds if we replaced $L=22.5 \mathrm{~cm}$ with $L^{\prime}=20 \mathrm{~cm}$; this $10 \%$ deviation shows that defining the particle flow rate as volume averages or fluxes does not lead strictly to the same values. Note that we also ran experiments with various $L$ values; measuring the intercorrelation functions of $N(t)$ measured in neighboring windows confirmed that $\nu_{\text {out }}$ is related to particle velocity [Böhm et al., 2004].

[35] We can compare the approximate solution (34) with the exact solution (1) to the master equation (20) for steady state conditions. For large systems $(L \rightarrow \infty)$, we have $\alpha=$ $\sigma+\nu_{\text {out }} \rightarrow \sigma$ while $\beta=\lambda+\nu_{\text {in }} \rightarrow \lambda$, which means that the variances and means of both distributions become closer. We retrieve the fact that in the large-system limit, a negative binomial distribution can be approximated by a normal distribution, with the only difference that normal distributions are continuous real-valued functions (which can then take negative values) whereas negative binomial distributions are functions that take nonnegative integer values. Note also that when $\mu>0$ (collective entrainment), the ratio $\operatorname{var} N / \bar{N}$ 


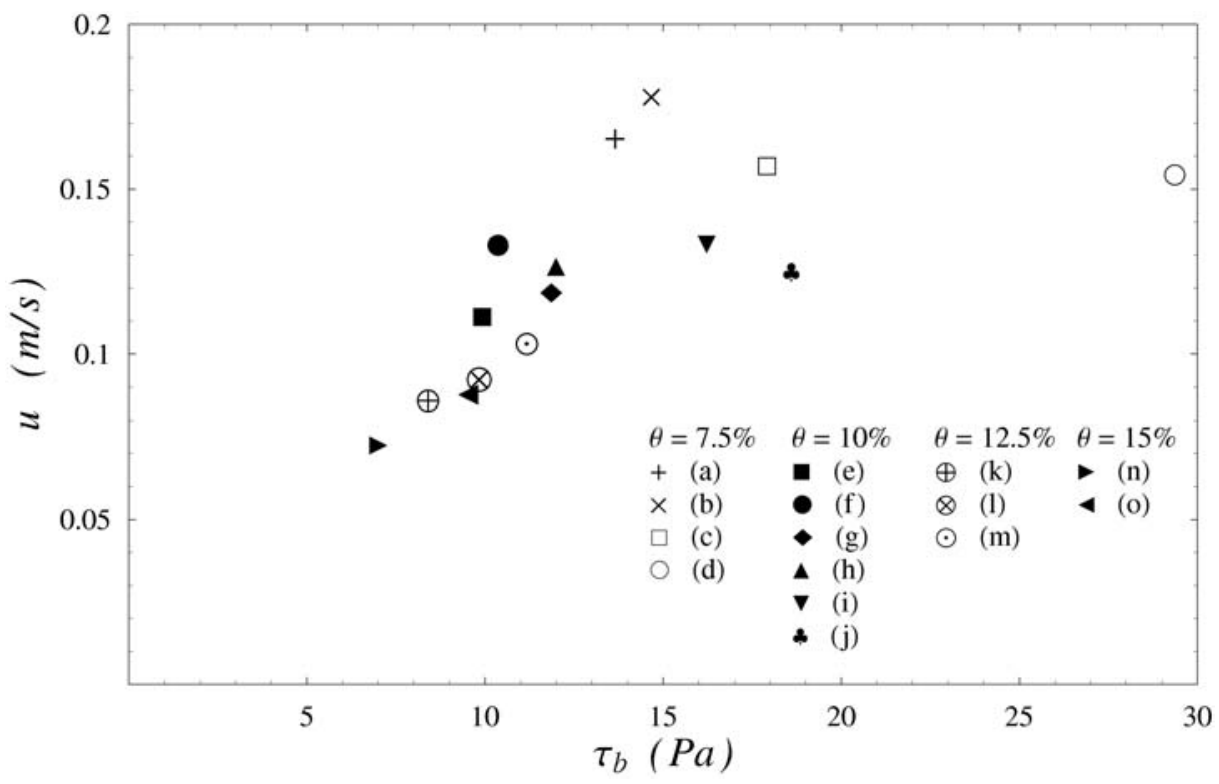

Figure 4. Relation between the bottom shear stress $\tau_{b}$ and the mean particle velocity $\bar{u}_{p}$.

always exceeds unity, which implies that large and frequent fluctuations occur. When $\mu=0$ (no collective entrainment), this ratio is unity and we retrieve the usual Poissonian limit of Einstein-like models. The autocorrelation time of the exact solution (1) to the master equation (20) is $t_{c}=(\alpha-\mu)^{-1}$ [Ancey et al., 2008]; in the large-system limit, we also find that the relaxation time approaches the autocorrelation time: $t_{r} \rightarrow t_{c}$.

[36] Figure 6 shows the probability distribution function of the number of moving particles $N$. We report the empirical distribution (dots), the exact theoretical solution (1) (dashed line), and the approximate analytical solution (34) (solid line).
The parameters of the distributions were estimated using the measured values var $N$ and $\bar{N}$ reported in Table 1; for the sake of readability, we plotted the discrete probability mass functions as continuous curves. First note the fairly good agreement between the data and theoretical probability distribution for all experiments. Local departures and data scattering are seen, but they are usually associated with low probabilities. Since 8000 data points were used for each run, empirical probabilities lower than $10^{-3}$ are unimportant. The plots confirm that the negative binomial distribution is a good candidate for describing the statistical behavior of $N$ over a wide range of flow conditions.

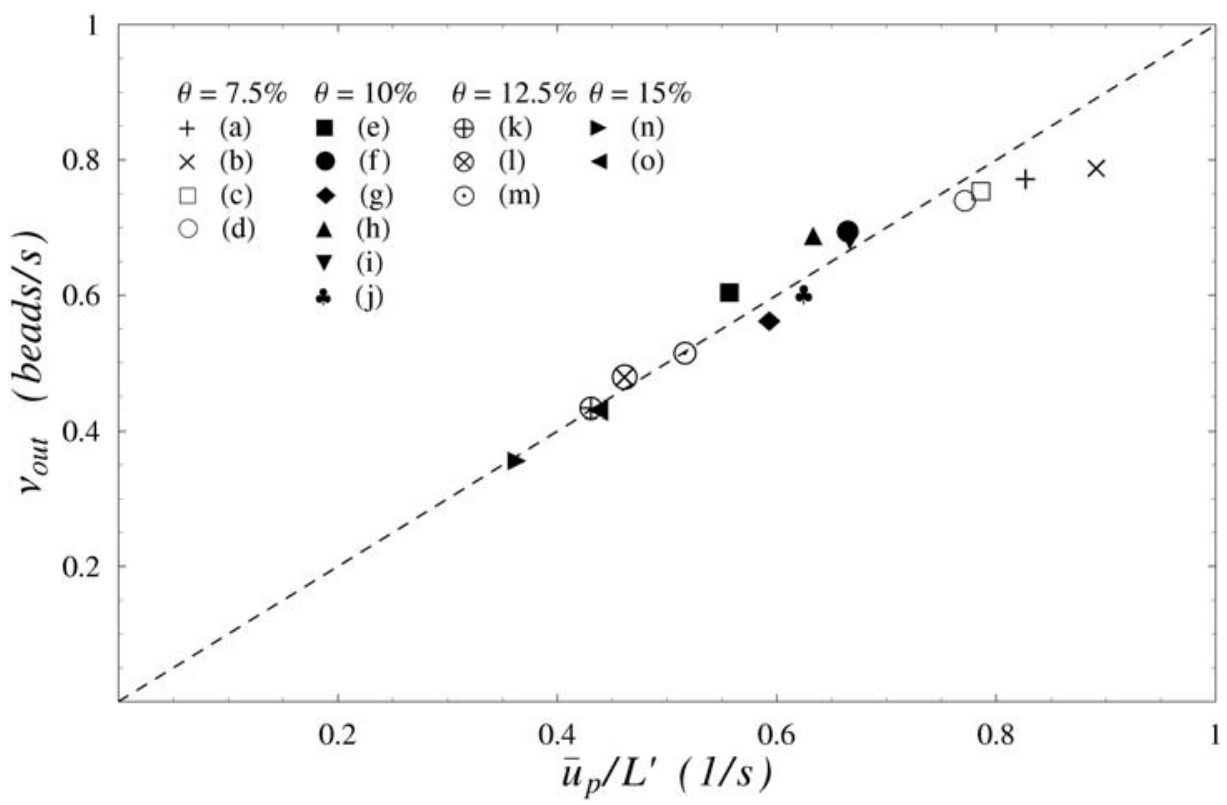

Figure 5. Relation between the measured $\nu_{\text {out }}$ values and the ratio $\bar{u}_{p} / L^{\prime}$ with $L^{\prime}=20 \mathrm{~cm}$. 

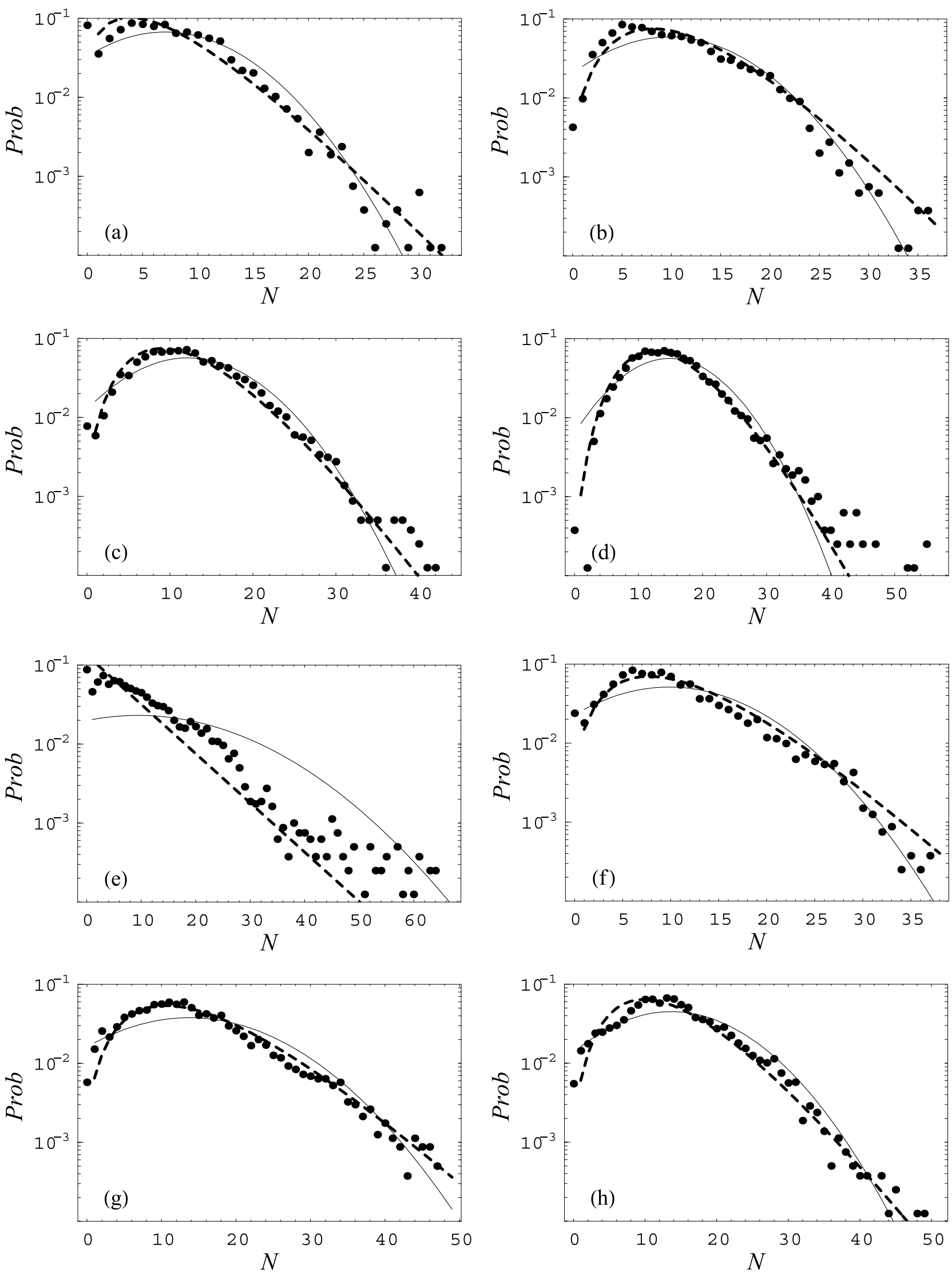

Figure 6. (a-o) Empirical probability density of the total number of moving beads $N$ (black dots). The dashed line is the probability density function of the negative binomial distribution. Experiments a-o. The solid line represents the Gaussian approximation (34). 

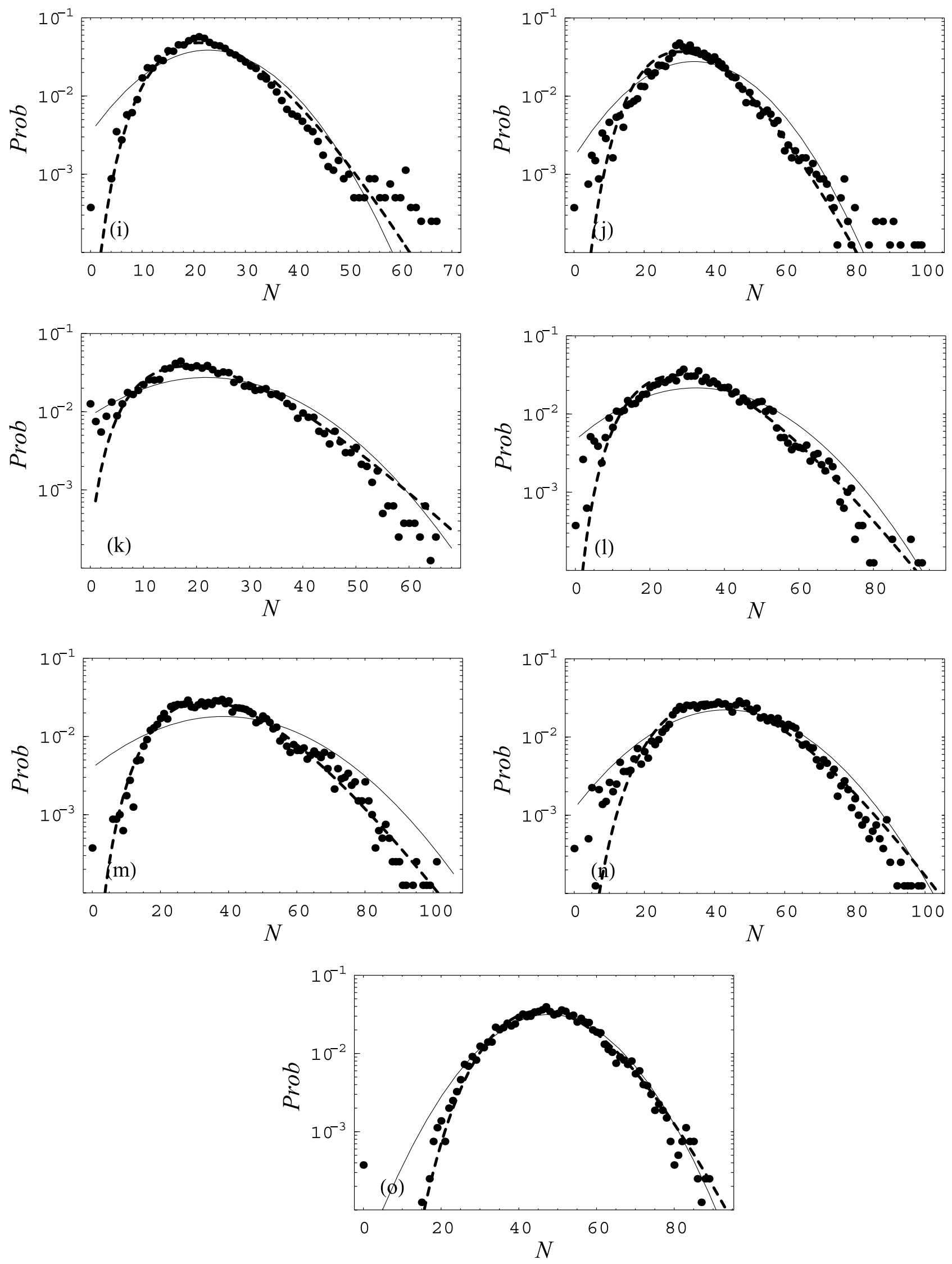

Figure 6. (continued) 
[37] There is a significant change in the distribution shape when the water discharge increases. When it is low (e.g., Figure 6e), the empirical distribution is close to a straight line on a logarithmic scale, implying that the number of particles decays exponentially within the observation window. For experiment e, the discrepancy between empirical and theoretical probability distributions is the most pronounced of all runs, which may mean that theory performs less well for these low discharges. With increasing water discharge, the probability distribution takes a bell shape, which is first skewed, then nearly symmetric at the highest slopes and flow rates (see Figure $6 \mathrm{n}$ ). In the case of run $\mathrm{n}$, the discrete empirical probability distribution can be closely approximated by a Gaussian distribution, as expected.

[38] For each run, the sample variance exceeded the mean, but the ratio var $N / \bar{N}$ decreased with increasing water discharge. For instance, for slope $\tan \theta=0.1$, the ratio $\operatorname{var} N / \bar{N}$ was as high as 7 for run e and dropped to below 3 for run 0 . Thus for each run, the number of moving particles varied frequently and widely, but with increasing water discharges, wide fluctuations became less frequent, which substantiates the idea that at high water discharges, sediment transport becomes more continuous. The normal distribution closely approximates the empirical distribution of $N$ when $N$ is sufficiently large (typically for $\bar{N}$ as large as 40 ). There is, however, no precise criterion that can serve to mark the lower bound from which this approximation holds.

\subsection{Implication for the Exner Equation}

[39] The stochastic Exner equation is given by (19), where the entrainment and deposition parameters $\sigma, \mu$, and $\lambda$ depend on the bottom shear stress (15) while the number of moving particles $\tilde{N}$ is the solution to the Ito differential equation (27). The full problem (19), (15), and (27) is prohibitively complex to be solved analytically because of nonlinearities arising in the coupled equations and stochastic fluctuations. Analytical approximations are available if we assume that the flow depth is nearly constant, an assumption which is quite common for planar bed lower-regime flow conditions [Exner, 1925; Jerolmack and Mohrig, 2005]. In that case, the bottom shear stress equation is constant: $\tau_{b}=\rho g h \sin \theta$.

[40] Different levels of approximation can be used to shed light on the behavior of the system of equations (19) and (27). The crudest approximation is to assume that $N$ varies so fast with time that for periods of time longer than the relaxation time $t_{r}$, the number of moving particles reaches a quasi-stationary state in which $d N / d t \rightarrow 0$ (or $d \eta_{1} \rightarrow 0$ ). In this case, using (28) and (32), we obtain

$$
\tilde{N}=\frac{\tilde{\lambda}}{\sigma-\mu}+\frac{\sqrt{\tilde{B}\left(n_{*}\right)}}{\sigma-\mu} \frac{d \mathcal{W}}{d t},
$$

which results in

$$
b(x, t)=\sqrt{\tilde{B}\left(n_{*}\right)} \mathcal{W}(t) .
$$

This equation shows that the probability distribution function of bed elevation $b$ is a Gaussian distribution; furthermore, the variance of $b$ grows linearly in time since the variance of $\mathcal{W}$ grows like $t$. A Gaussian distribution has often been found to be a correct approximation of bed elevation profiles [Nikora et al., 1998]. Experimental measurements provided variance growing as a power function of time, with an exponent ranging from 0.56 to 1.36 depending on particle size [Coleman et al., 2005]. In spite of its crudeness, this approximation provides a reasonably correct description of the bed form evolution for planar bed incipient motion flow conditions.

[41] In the large-system limit $(L \gg 1)$ and slightly bumpy bed $\left(\partial_{x} b \ll \tan \theta\right)$, another approximation consists in expanding $b$ into an $\epsilon$ power series as we did for $N: b(x, t)=$ $b_{0}(x, t)+\epsilon b_{1}(x, t)+\cdots$. We then obtain a hierarchy of equations, where the exchanges rates $\sigma, \mu$, and $\tilde{\lambda}$ are constant. To zeroth order, we obtain

$$
\psi \frac{\partial b_{0}}{\partial t}=(\sigma-\mu) \tilde{n}_{*}-\tilde{\lambda} .
$$

Since $N_{*} \rightarrow N_{\infty}=\tilde{\lambda} /(\sigma-\mu)$ when $t \rightarrow \infty$, then we get $\partial_{t} b \rightarrow 0$, which means that at order $\epsilon^{0}$, the bed elevation reaches a constant level. To first order, we obtain

$$
\psi \frac{\partial b_{1}}{\partial t}=(\sigma-\mu) \eta_{1},
$$

with $\eta_{1}$ solution to (32) subject to the initial condition $\eta_{1}(0)=0$ :

$$
\eta_{1}(t)=\int_{0}^{t} \exp \left(-\left(t-t^{\prime}\right) / t_{r}\right) \tilde{B}^{1 / 2}\left(n_{*}\left(t^{\prime}\right)\right) d \mathcal{W}\left(t^{\prime}\right),
$$

from which we deduce that

$b_{1}(x, t)=(\sigma-\mu) \int_{0}^{t} d t^{\prime} \int_{0}^{t^{\prime}} \exp \left(-\left(t^{\prime}-t^{\prime \prime}\right) / t_{r}\right) \tilde{B}^{1 / 2}\left(n_{*}\left(t^{\prime \prime}\right)\right) d \mathcal{W}\left(t^{\prime \prime}\right)$.

At long times, we can compute the first moments of $b_{1}$ : $\left\langle b_{1}\right\rangle=0$ and $\left\langle b_{1}^{2}\right\rangle=\tilde{B}\left(N_{\infty}\right) t$, which is consistent with the crude approximation (36). In particular, we retrieve that the variance varies linearly with time on the long range. Another interesting point is related to the spectral density function $\mathcal{S}(\omega)$ of $\tilde{n}_{1}$ : since on the long term $\eta_{1}$ is an Ornstein-Uhlenbeck process with constant parameter $k_{o}=$ $\sigma-\mu$ and diffusion $D_{o}=\tilde{B}\left(n_{\infty}\right)$, the spectrum of $\tilde{N}$ is [Gardiner, 1983]

$$
\mathcal{S}(\omega)=\frac{\tilde{B}\left(N_{\infty}\right)}{2 \pi} \frac{1}{\omega^{2}+(\sigma-\mu)^{2}},
$$

with $\omega$ the angular frequency. This shows that the spectrum tends toward a plateau when $\omega \rightarrow 0$ and decreases as $\omega^{-2}$ in the limit of $\omega \rightarrow \infty$. The bed elevation following the same trend, we retrieve the $\omega^{-2}$ decay observed by Nikora et al. [1997] in the high-frequency range, but the low-frequency behavior $\mathcal{S}(\omega) \propto \omega^{-3}$ is not retrieved. As discussed by Nikora et al. [1997], note that there is no consensus in the literature about the decay rate of $\mathcal{S}$ in the high-frequency domain (some authors providing $\mathcal{S} \propto \omega^{-3}$ ). 
[42] The last point concerns the variation in the root mean square of bed elevation $w$ with length $L$. Since $b=$ $b_{0}+\epsilon b_{1}+\cdots$ with $\epsilon=L^{-1 / 2},\left\langle b_{1}\right\rangle=0$, and $\left\langle b_{1}^{2}\right\rangle=\tilde{B}\left(N_{\infty}\right) t$, we have

$$
w(L, t)=\left(\frac{1}{k} \sum_{i=1}^{k}\left(b\left(x_{i}, t\right)-\bar{b}\right)^{2}\right)^{1 / 2}=\epsilon\left(\frac{1}{k} \sum_{i=1}^{k} b_{1}^{2}\left(x_{i}, t\right)\right)^{1 / 2},
$$

where $b\left(x_{i}, t\right)$ denotes the bed elevation at a site $x_{i}$ and time $t, k$ is the number of sites over the length $L$, and $\bar{b}$ is the average of $b$ over the domain of length $L$. The sum of $k$ square Gaussian variables of mean 0 and constant variance (at time $t$ ) is a (noncentral) chi-square distribution with mean $k$. Since $k \propto L$, we then deduce that $w(L, t) \propto \epsilon L=L^{1 / 2}$. This is roughly consistent with the results obtained by Jerolmack and Mohrig [2005], who obtained $w \propto L^{0.64}$; although there is no perfect match, both values are reasonably close (given that the data span over a limited range of variations).

\subsection{Numerical Simulations}

[43] Two types of numerical models can be developed depending on whether $N$ is considered a continuous or discrete random variable. The former type is the easiest to implement since it involves solving a set of coupled firstorder differential equations including a stochastic differential equation; there are many relatively simple, but fairly efficient algorithms for that purpose [Higham, 2001]. The latter type is a little more delicate to use in that it is based on exact or approximate stochastic simulation algorithms, whose running is known to be fairly slow [Gillespie, 2007].

[44] Here we address the case in which the flow depth is nearly constant and $\tilde{N}$ is considered a continuous variable. We have to solve (19), (15), and (27). We use a first-order explicit forward Euler scheme (a variant called EulerMaruyama method) to discretize the governing equations with a constant time step $\Delta t$ [Iacus, 2008]. The spatial domain of length $L$ is divided into $S$ cells centered at nodes $x_{i}=\left(i-\frac{1}{2}\right) \Delta x(1 \leq i \leq S)$ of size $\Delta x$. The bed elevation is evaluated at each node $x_{i}$ and time $t_{k}=k \Delta t$, where $\Delta t$ is a very small time increment. At time $t_{k_{+1}}=(k+1) \Delta t$, we have

$$
\begin{gathered}
\tilde{N}_{i, k+1}=\tilde{N}_{i, k}+\Delta t \tilde{A}\left(\tilde{N}_{i, k}\right)+\zeta \sqrt{\Delta t \tilde{B}\left(\tilde{N}_{i, k}\right)} \\
b_{i, k+1}=b_{i, k}+\frac{\Delta t}{\psi}\left(\left(\sigma+\nu_{\text {out }}-\mu\right) \tilde{N}_{i, k}-\tilde{\lambda}\left(\tau_{i, k}\right)\right) .
\end{gathered}
$$

where $\zeta$ is picked from a Gaussian distribution with mean 0 and variance 1 . This scheme is first order in time and requires tiny time steps to be accurate; there are other methods that perform better and converge faster [Rümelin, 1982]. Typically, with the Euler-Maruyama scheme, the simulation of a $60 \mathrm{~s}$ run requires computational times as long as $6 \mathrm{~h}$ on a standard personal computer.

[45] To illustrate this scheme, we present the results of a numerical simulation for a virtual $25 \mathrm{~m}$ long flume tilted at $\theta=5^{\circ}$ to the horizontal. The parameters of the simulation are reported in the caption of Figure 7. As shown in Figure 7a, the solid concentration varies substantially along the bed, causing large variations in the bed elevation (see Figure 7b). The empirical distribution of the simulated $N$ samples are nearly Gaussian (see Figure 7d), while the bed elevation sample is also Gaussian (see Figure 7c)with a variance that grows nearly linearly with increasing time (see Figure 7e). These results are in good agreement with the analytical approximations seen in section 3.2.

\section{Concluding Remarks}

[46] In this paper we have built a reasonably simple framework for simulating the bed evolution of a river bed made up of coarse sediment when particles move sporadically along the bed. In a first-order approximation of lower-regime flow conditions, we ignore the details of fluid flow and focus our attention on the sediment-stream interface. The model is thus a first stab at modeling bed load transport when there is no significant feedback from water flow.

[47] Our posit is that for bottom shear stresses just in excess of the threshold of incipient motion (i.e., for transport stage parameters $T<3$ ), sediment flux is driven by the concentration in moving particles rather than particle velocity (which varies weakly with increasing bottom shear stress). Therefore, to compute the solid discharge or track the bed evolution, it is fundamental to predicting how the number of moving particles varies with time. With this objective in mind, we idealized particle entrainment and deposition as population exchanges between the stream and the bed inside a finite control volume. We used an immigration-emigration birth-death Markov process to theoretically describe the probabilities that particles are entrained from or deposited on the bed, while other particles may enter or leave the control volume. In an earlier paper, Ancey et al. [2008] showed that under steady state conditions, the probability distribution of the number of moving particles is a negative binomial distribution. This distribution has interesting properties such as its wide variance, which may explain the huge fluctuations experienced by sediment flux, and thus is an interesting alternative to more elaborated formalisms (based on fractional diffusion) presented in this special issue. In this paper, we extend theory by considering the limit of large systems. When the size of the control volume or the number of moving particles becomes large (typically $\bar{N} / L>2$ particles/cm), we show that the concentration in moving particles behaves like a Gaussian random variable. This approximation is here particularly helpful in that it transformed the Exner equation (14) into an Ito stochastic differential equation (14). Some interesting approximations can be directly inferred from this equation. In particular, under planar bed lower-regime conditions, it can be shown that the bed elevation density distribution is Gaussian, with a variance growing linearly with time. Both analytical approximations and numerical simulations support the idea developed by Jerolmack and Mohrig [2005] according to which accounting for fluctuations of sediment flux in the Exner equation is crucial to obtaining bed topographies comparable to natural river beds.

[48] There are several related topics that would deserve further attention. First, as noted in Appendix A, there are several ways of defining the solid discharge and it is currently quite unclear whether these definitions somehow overlap and match. In particular, the statistical properties of the solid discharge depend a great deal on the definition used. A point 

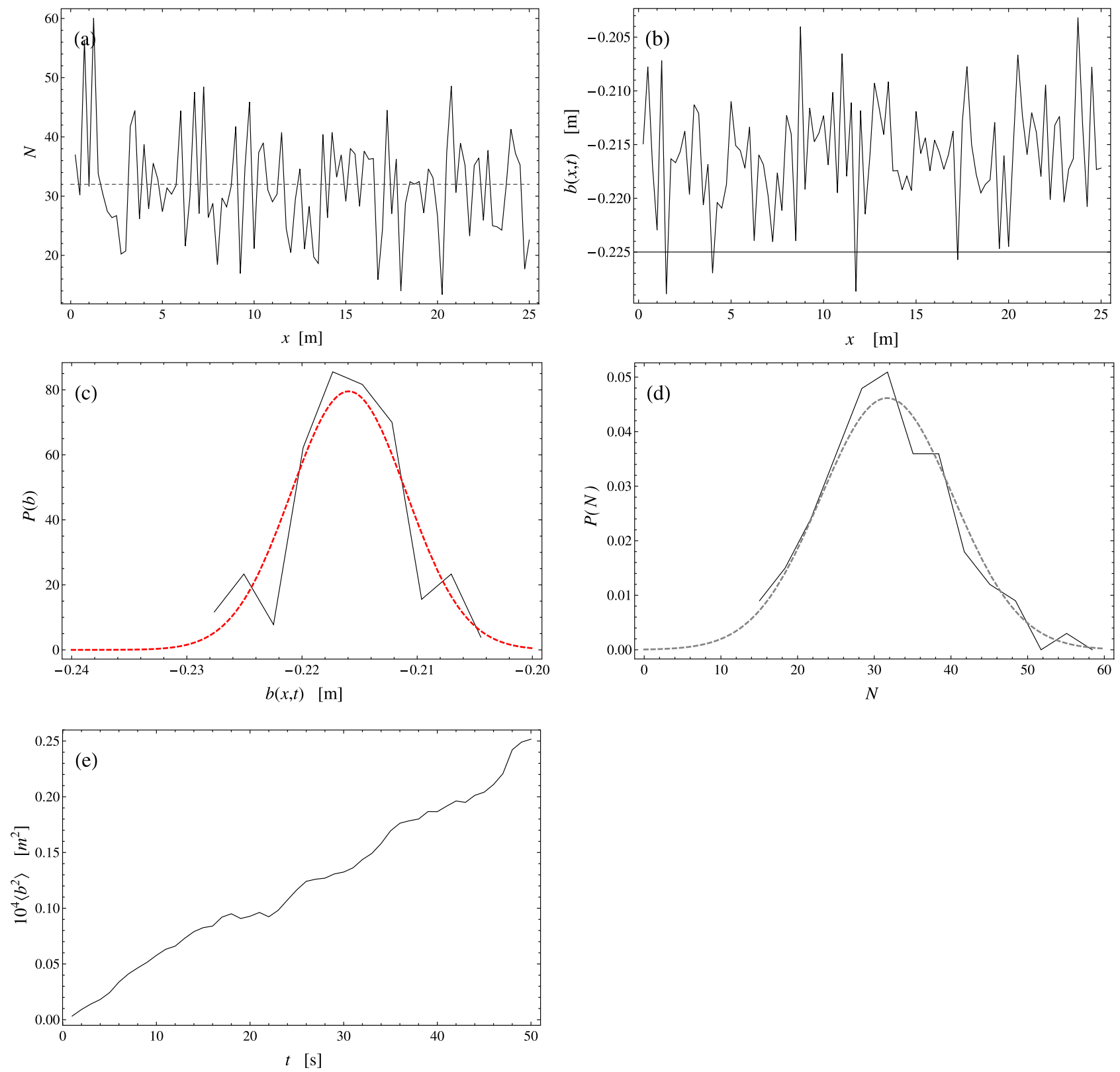

Figure 7. Numerical results $\Delta x=0.25 \mathrm{~m}$ and $\Delta t=10^{-4} \mathrm{~s}$; the exchange rates are grossly estimated from our experiments (see Appendix A): $\lambda\left(\tau_{b}\right)=2\left(\tau_{b}-7\right)($ beads $/ \mathrm{s}), \mu\left(\tau_{b}\right)=4(1 / \mathrm{s}), \sigma\left(\tau_{b}\right)=5(1 / \mathrm{s})$, $\nu_{\text {in }}\left(\tau_{b}\right)=5+\lambda\left(\tau_{b}\right) / 3$ (beads $\left./ \mathrm{s}\right)$, and $\nu_{\text {out }}\left(\tau_{b}\right)=\tau_{b} / 20(1 / \mathrm{s})$. The particle radius is $a=3 \mathrm{~mm}$. (a) Variation of $N$ along the flume at time $t=50 \mathrm{~s}$. (b) Bed elevation profile at time $t=50 \mathrm{~s}$. (c) Empirical probability distribution of $b$ as well as the fit by a normal distribution (dashed line) at $t=50 \mathrm{~s}$; the empirical distribution was built using the same data as those reported in Figure 7b. (d) Empirical probability distribution of $N$ as well as the fit by a normal distribution (dashed line) at $t=50 \mathrm{~s}$; the empirical distribution was built using the same data as those reported in Figure 7a. (e) Evolution of the bed elevation variance.

that requires more consideration is the relation between the solid discharge $q_{s}$, mean particle velocity $\bar{u}_{p}$, and particle concentration $\tilde{N}$. Here, relying on laboratory observations, we assumed that much of the $q_{s}$ variance is due to variations in the particle concentration rather than particle velocity; this contrasts with what is assumed in most theoretical bed load transport models. Second, the statistics of bed load transport is substantially influenced by the scale of observation (i.e., the length $L$ of the control volume), with the important con- sequence that the bed elevation features are also scaledependent, as noted by Jerolmack and Mohrig [2005]. For short spatial scales, the number of moving particles resembles a discrete and random process, while for long scales, the behavior is more continuous and the system exhibits Gaussian fluctuations. Note that even on small spatial scales $(L \approx 40 a$, with $a$ the particle radius), the particle concentration can exhibit Gaussian behavior when the particles move in large numbers in the control volume. Third, in contrast with solute 
transport in porous media (see the discussion in section 2.2), there is still no clear connection between Eulerian and Lagrangian descriptions, which would be essential for both theoretical and practical reasons.

[49] A strength of the theoretical model is its versatility, which should allow consideration of scenarios much closer to reality than the one briefly presented in section 3.6 and extension to similar problems, such as long-term behavior of rivers [Tipper, 2007] and landforms [Turcotte, 1995]. The model offers a simple and efficient way of describing complex issues in sediment transport by avoiding some of the impediments encountered with other approaches. For instance, detailed experimental investigations have revealed that instantaneous forces experienced by coarse particles lying on the bed are not strongly correlated with the mean velocity field of the flow [Schmeeckle et al., 2007]. This suggests that a fully deterministic approach to sediment transport remains a formidable challenge; our hybrid stochastic-physical approach tries to get around this difficulty by substituting the complex coupling between flow and sediment with a statistical description. Another great advantage is that all parameters used in the model are physical quantities that can be fairly easily measured in the laboratory or in the field using image processing [Drake et al., 1988; Ancey et al., 2008]. There are a few limitations of this approach that come out. First, when there is no coupling between the water flow and sediment transport (as assumed in the numerical simulations presented in section 3.6), the model predicts bed roughness growing like $t^{1 / 2}$. There is a naturally an upper bound to this growth imposed by the water flow [Coleman et al., 2005], in particular by coherent turbulent structures that are generated by bed forms, interfere with the bed interface, and affect turbulence production/dissipation processes [Best, 2005]. Second, numerical methods for solving stochastic problems require considerable amounts of computational times and are not free of pitfalls. In particular, there is no available analytical solution against which numerical models can be checked.

\section{Appendix A: Computation of the Solid Discharge}

[50] There is no unique way to define the solid discharge. The random and time-evolving nature of solid discharge raises the problem of a suitable statistical procedure that enables computation and description of sediment flux. In spite of valuable efforts in recent years to gain insight into this issue, it is still unclear whether the different solid-discharge forms lead to compatible results in terms of statistical properties. Here we review the main forms that are currently of common use and comment on their strengths and weaknesses.

[51] While theoretically the volume solid discharge can always be defined as the flux of particles through a cross section $S$ of unit width:

$$
q_{s}=\int_{S} \mathbf{u}_{p} \cdot \mathbf{k} d S,
$$

with $\mathbf{u}_{p}$ the particle velocity field and $\mathbf{k}$ the unit normal to $S$, this definition is rarely used in practice as it is more suited to continuous fields than discrete elements; the dimension of $q_{s}$ is $\mathrm{m}^{2} \mathrm{~s}^{-1}$. At least four different forms of the solid discharge have been proposed for practical or theoretical purposes.
They all assume bed load at equilibrium or near equilibrium. On average, they may provide the same values, but the statistical properties of $q_{s}$ are influenced a great deal.

[52] A variant of equation (A1) is to count the number of particles that passed through $S$ over a short time increment $\delta t$. The main problem is that $q_{s}$ is a step function, which takes zero values except at the times of arrival of individual particles; the resulting signal is then very noisy. In practice, using hydrophones or piezoelectric sensors makes it possible to evaluate $q_{s}$ by correlating acoustic signals (or vibrations) and sediment rate [Bänziger and Burch, 1991; Carling et al., 1998; Tunnicliffe et al., 2000; Rickenmann and McArdell, 2007, 2008; Turowski et al., 2008]. Another related form is to count the number of particles that arrive up to time $t$ or to integrate $q_{s}$ over a short period of time. In the laboratory, this is done by weighting the material accumulated in a basket located at the flume outlet while in the field, sediment traps and bed load samplers are used. These techniques do not provide $q_{s}$ directly, but the sediment volume per unit width $V(t)=\int q_{s}(t) d t$. In principe, it should be possible to differentiate $V$ to derive $q_{s}$, but in practice, fluctuations in the $V(t)$ records make this operation delicate, which explains why sampling time is a key issue when trying to properly evaluate the solid discharge [Bunte and Abt, 2005; Singh et al., 2009].

[53] Another approach was taken by Einstein [1950] according to whom sediment transport does not result from an equilibrium in the momentum transfers between solid and liquid phases, but rather from the difference between the entrainment and deposition rates, $E$ and $D$, respectively, which are functions of the flow conditions and bed geometry [Einstein, 1950]. This amounts to writing that on a small interval $\Delta x$, the solid discharge variation is $\delta q_{s}=(E-D) \Delta x$, and so the solid discharge at bed equilibrium is the implicit solution to the equation $E=D$. Einstein found that the sediment transport rate is

$$
q_{s}=E \ell_{s},
$$

where $\ell_{s}$ is the mean length traveled by individual particles during each step. Several field measurement campaigns used Einstein-like definitions to monitor bed load transport under incipient motion conditions [Drake et al., 1988; Habersack, 2001; Wilcock, 1997; Pryce and Ashmore, 2003]. Laboratory experiments as well as theoretical analyses made extensive use of this definition [Fernandez Luque and van Beek, 1976; Seminara et al., 2002]. The variance of $q_{s}(t)$ records results from both fluctuations in entrainment rates $E$ and heap lengths $\ell_{s}$.

[54] The use of tracer stones in gravel bed rivers has given rise to a third relation. From the observation that particles can be moving, lying at rest on the bed surface, or buried in the bed, one can define a virtual velocity, which is the timeaveraged velocity $U_{p}$ (called virtual particle velocity) of a single particle regardless of its state. Only the upper bed layer participates in bed load transport and is therefore termed the active layer; the thickness of this layer is $L_{a}$ and represents the depth down to which the bed is continuously reworked by fill and scour. Mass conservation then implies that

$$
q_{s}=U_{p} L_{a} .
$$




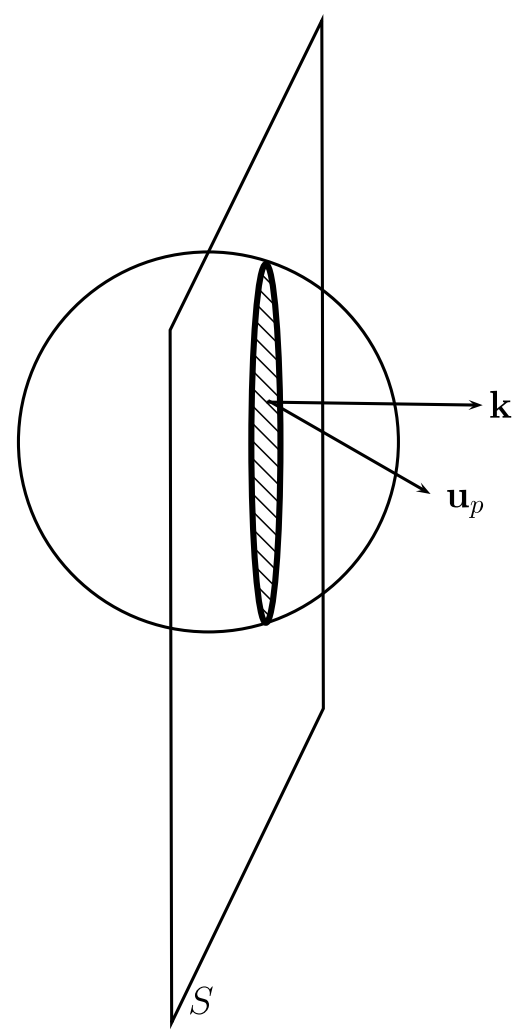

Figure A1. Sketch showing the flux of particles through $S$.

The use of this equation has been documented for both natural rivers [Ferguson et al., 2002; Ferguson and Hoey, 2002; DeVries, 2002; Cudden and Hoey, 2003] and flume experiments [Wong et al., 2007]. The statistical properties of $q_{s}(t)$ depend on the fluctuations of $U_{p}$ and $L_{a}$, which are little known in practice.

[55] Taking inspiration from kinetic theories of gas and two-phase flow rheology [Drew and Passman, 1999; Lhuillier, 1992], we can define the solid discharge as the ensemble average of the particle flux

$$
\left\langle q_{s}\right\rangle=\int_{S} \int_{R^{2}} P\left[\mathbf{u}_{p} \mid \mathbf{x}, t\right] \mathbf{u}_{p} \cdot \mathbf{k}|d \mathbf{x}| d \mathbf{u}_{p},
$$

where $P\left[\mathbf{u}_{p} \mid \mathbf{x}, t\right]$ is the probability that a particle crosses the control surface $S$ at position $\mathbf{x}$ and time $t$ with velocity $\mathbf{u}_{p}$. Note that $\mathbf{u}_{p}$ is a velocity field, which is $\mathbf{u}_{p}(\mathbf{x}, t)=\mathbf{u}_{G}+\Omega \times$ $\left(\mathbf{x}-\mathbf{x}_{G}\right)$ where $\mathbf{x}$ lies inside a particle with velocity $\mathbf{u}_{G}$ at the center of mass $\mathbf{x}_{G}$ and rotational velocity $\Omega$ when $\mathbf{x}$ lies in the fluid phase, this field is zero. Figure A1 shows a particle crossing the control $S$.

[56] Under near-equilibrium conditions, ensemble averages can be swapped with volume averages; equation (A4) can then be recast in the following form

$$
\left\langle q_{s}\right\rangle=\int_{b}^{s} c(y) u_{p}(y) d y
$$

where we retrieve the correspondence between concentration and probability of finding one particle at a given place (see section 2.2). This equation was used notably by Wiberg and Smith [1989]. Similar expressions were also used by Bridge and Dominic [1984], Yalin [1963], and Kovacs and Parker [1994]. The equivalence between ensemble and volume averages makes sense when the entire flow is homogeneous, i.e., when the particles are homogeneously distributed in the streamwise direction. For two-phase flows over mobile beds, bed forms usually affect the distribution of moving particles, which makes the assumption of homogeneity dubious. A number of tricks have been developed to extend ensemble and volume average techniques to nonuniform particle suspensions; for instance, an infinite uniform suspension can be approximated by the periodic repetition of a control volume. Numerically, this amounts to carrying out the simulation in a finite box with periodic boundary conditions [Marchioro et al., 2000]. It is convenient to introduce a finite volume average solid discharge

$$
\left\langle q_{s}\right\rangle_{L}=\frac{S}{V} \sum_{i=1}^{N} u_{i} v_{p}=\frac{v_{p}}{L} \sum_{i=1}^{N} u_{i},
$$

where $v_{p}$ is the particle volume and integration has been performed over the control volume $(\mathcal{V}=L \times S)$ of length $L$, which is sufficiently long to contain a number of particles, but is short enough compared to the scale of variation of $q_{s}$ on the macroscopic scale. We let $u_{i}=\mathbf{u}_{p} \cdot \mathbf{k}$ denote the streamwise velocity component of particle $i$. Here we thus define the particle flow rate $\dot{n}=Q_{s} / v_{p}$ as

$$
\dot{n}=\frac{1}{L} \sum_{i=1}^{N} u_{i}=\frac{N}{L} \bar{u}_{p},
$$

where $\bar{u}_{p}$ denotes the mean particle velocity.

\section{Appendix B: Birth-Death Model}

[57] To begin with, we set the scene by developing an analogy between chemical reactions and particle transport in streams. This makes it possible to use many of the computational tools developed in chemical kinetics. We then outline the stochastic basis of the model.

[58] As depicted in Figure 1, we consider a control volume $\mathcal{V}$ (per unit width) within which we track moving particles and record deposition/entrainment events. A few solid particles are entrained by the water stream: they can roll/slide along the bed or they can leap and stay in saltation in the water stream for short time periods. We refer to the former motion as the rolling regime and to the latter as the saltating regime. As we are especially interested in low bed load transport, we stress flows with a fairly low fluid velocity: the trajectory of a single particle then exhibits a succession of rests and moves in a rolling or saltating regime. Below we do not discriminate between rolling and saltation and treat both motions as a single "chemical" species which we call the moving particles (M). We assume that the number of particles (B) making up the bed is infinite, i.e., whenever a particle at the bed interface is set in motion, the shape of the interface is altered, but not the number of particles available to entrainment at the bed interface.

[59] Let us write the exchanges between the bed and the stream like chemical equations. 
[60] 1. A moving particle $M$ can come to rest (at rate $\sigma_{0}$ ) and thus be transformed into a bed particle $B$

$$
M \rightarrow B \text {. }
$$

[61] 2. The trajectory of a moving particle $M$ can be disturbed by a bed particle, transforming $M$ into a bed particle $B\left(\right.$ at rate $\left.\sigma_{1}\right)$

$$
M+B \rightarrow B+B
$$

[62] 3. A moving particle can interfere with a bed particle and entrain it (at rate $\mu$ )

$$
M+B \rightarrow M+M
$$

[63] 4. A bed particle can be entrained by the fluid and set in motion (at rate $\lambda$ )

$$
B \rightarrow M
$$

[64] Pursuing our analogy with chemical systems, we denote the mean number of moving particles by $[M] . B$ is like a catalyst: it is needed in the transformation, but its mean number does not vary with time; that is, $[B]$ is fixed. The resulting reaction rate equation is

$$
\frac{d}{d t}[M]=\lambda+\mu[M]-\sigma[M],
$$

with $\sigma=\sigma_{0}+\sigma_{1}$. As seen in (B5), we have no means of differentiating the different deposition processes $\sigma_{1}$ and $\sigma_{0}$, whereas entrainment includes both individual entrainment events $(\lambda)$ and collective effects $(\mu)$, a point that is essential to explaining the appearance of wide fluctuations, i.e., nonGaussian fluctuations of sediment transport rate [Ancey et al., 2008]. We can now place this model on a firmer theoretical ground by describing the statistics of particle exchanges. Note also that since we are counting particles in a finite and open control window $\mathcal{V}$, we must take into account that particles can enter and leave $\mathcal{V}$ at any time. Inflow and outflow rates are denoted by $\nu_{\text {in }}$ and $\nu_{\text {out }}$, as shown in Figure 1 . An earlier paper gave further information on the physical mechanisms [Ancey et al., 2008].

[65] The model we have developed belongs to the class of Markov process with discrete states in continuous times since $N(t)$ is an integer-valued function of time; more precisely, we describe sediment transport using an emigrationimmigration birth-death process [see Cox and Miller, 1965, chapter 5]. We consider the following exchanges over the time increment $\Delta t$, which is assumed to be sufficiently small that two events cannot occur in $(t, t+\Delta t)$.

[66] 1 . Beads enter the window at rate $\nu_{i n}$ (immigration). The probability that the number of particles in $\mathcal{V}$ is incremented by one is then

$$
P(n \rightarrow n+1 ; \Delta t)=\nu_{\text {in }} \Delta t+o(\Delta t) .
$$

As explained in section 2, the probability that more than one particle arrive at the same time or within the time interval $[t, t+\delta t)$ is zero in birth-death models, which means physically that the particle flow is rather dilute and that sufficiently short time increments can be selected for this condition to be satisfied.
[67] 2. Moving particles leave the window independently at rate $\nu_{\text {out }}$ (emigration). Since there are $n$ particles inside $\mathcal{V}$, the transition probability is

$$
P(n \rightarrow n-1 ; \Delta t)=n \nu_{\text {out }} \Delta t+o(\Delta t) .
$$

[68] 3. Two processes enable entrainment of particles from the bed (birth): a particle can be dislodged from the bed by the water stream at rate $\lambda>0$ or a moving particle can destabilize a stationary one and set it moving. This occurs at rate $\mu$ for any moving particle within the observation window. The corresponding transition probabilities are

$$
\begin{aligned}
& P(n \rightarrow n+1 ; \Delta t)=\lambda_{1} \Delta t+o(\Delta t) \text { and } \\
& P(n \rightarrow n+1 ; \Delta t)=\mu n \Delta t+o(\Delta t),
\end{aligned}
$$

respectively.

[69] 4. A moving particle can come to rest within the window, independently at rate $\sigma$ for each moving particle (death). The transition probability is thus

$$
P(n \rightarrow n-1 ; \Delta t)=n \sigma \Delta t+o(\Delta t)
$$

[70] With these assumptions and the discrete ChapmanKolmogorov equation

$$
P(n ; t+\Delta t)=\sum_{-\infty}^{+\infty} P(n+i ; t) P(n+i \rightarrow n ; \Delta t),
$$

we obtain a set of equations [Ancey et al., 2008]

$$
\begin{aligned}
P(n ; t+\Delta t)= & \alpha(n+1) \Delta t P(n+1 ; t) \\
& +P(n-1 ; t)\{\beta+(n-1) \mu\} \Delta t \\
& +P(n ; t)\{1-\Delta t(\beta+n \alpha+n \mu)\}+o(\Delta t)
\end{aligned}
$$

for $n=1,2 \ldots$, and

$$
P(0 ; t+\Delta t)=\alpha P(1 ; t) \Delta t+P(0 ; t)(1-\beta \Delta t)+o(\Delta t),
$$

for $n=0$, with the shorthand notation $\alpha=\sigma+\nu_{\text {out }}$ and $\beta=$ $\lambda+\nu_{\text {in }}$. On rearranging the terms and letting $\Delta t \rightarrow 0$, we obtain the master equation

$$
\begin{aligned}
\frac{\partial}{\partial t} P(n ; t)= & (n+1) \alpha P(n+1 ; t)+(\beta+(n-1) \mu) P(n-1 ; t) \\
& -(\beta+n(\alpha+\mu)) P(n ; t) \\
\frac{\partial P(0, t)}{\partial t} & =\alpha P(1 ; t)-\beta P(0 ; t)
\end{aligned}
$$

[71] At time $t=0$, there are $N=N_{0}$ particles within the control volume, so we set

$$
P(n ; 0)=\delta\left(n-N_{0}\right),
$$

where $\delta$ is the Kronecker delta function. 


\section{Notation}

\section{Roman symbols}

$A$ drift function, see equation (20).

$a$ particle radius.

$b$ elevation of the bed surface.

$B$ diffusion function, see equation (20).

$c$ concentration.

$d$ distance traveled by particles.

$D_{o}$ diffusion coefficient in the Ornstein-Uhlenbeck process.

$D$ deposition rate in $\mathrm{m}^{3} \mathrm{~s}^{-1}$.

$E$ entrainment rate in $\mathrm{m}^{3} \mathrm{~s}^{-1}$.

$g$ gravity acceleration $g=9.81 \mathrm{~m} \mathrm{~s}^{-2}$.

$h$ flow depth.

$K$ diffusion coefficient in $\mathrm{m}^{2} \mathrm{~s}^{-1}$.

$k$ dummy variable.

k unit vector.

$k_{o}$ parameter in the Ornstein-Uhlenbeck process.

$L$ window length.

$\ell_{s}$ heap length.

$N$ number of particles within the window.

$n$

$N_{*}$

$N_{0}$

$\bar{N}$

$\dot{n}$

$\tilde{N}$ linear density of particles in beads $/ \mathrm{m}$.

$\tilde{N}_{\infty}$ asymptotic value of the linear density.

$\mathcal{N}$ Gaussian distribution.

$p$ parameter of the negative binomial distribution, see equation (1).

$P$ probability density function.

$\bar{Q}_{s}$ mean volume particle flow rate in $\mathrm{m}^{3} \mathrm{~s}^{-1}$.

$Q_{s}$ volume particle flow rate in $\mathrm{m}^{3} \mathrm{~s}^{-1}$.

$q_{s}$ volume particle flow rate per unit width in $\mathrm{m}^{2} \mathrm{~s}^{-1}$.

$q_{w}$ water flow rate per unit width in $\mathrm{m}^{2} \mathrm{~s}^{-1}$.

$r$ parameter of the negative binomial distribution, see equation (1).

$s$ elevation of the free surface.

$\mathcal{S}$ spectrum, see equation (41).

$S$ number of cells, see section 3.6.

$S$ cross section of the control volume, see Appendix A.

$t$ time.

$T$ transport stage parameter $T=\tau_{b} / \tau_{c}-1$.

$t_{c}$ autocorrelation time, see equation (3).

$t_{m}$ characteristic time of motion, see equation (3).

$t_{r}$ relaxation time, see equation (30) and section 3.3.

$u_{f}$ fluid velocity in $\mathrm{m} \mathrm{s}^{-1}$.

$\bar{u}_{p}$ mean particle velocity in $\mathrm{m} \mathrm{s}^{-1}$.

$u_{p}$ particle velocity in $\mathrm{m} \mathrm{s}^{-1}$.

$v_{p}$ particle volume in $\mathrm{m}^{3}$.

$\mathcal{V}$ control volume.

$\mathcal{W}$ Wiener process.

$W$ flume width.

$w$ root mean square of the bed elevation, see equation (42). $x \quad$ streamwise coordinate.

$y$ normal coordinate.

Greek and compound symbols

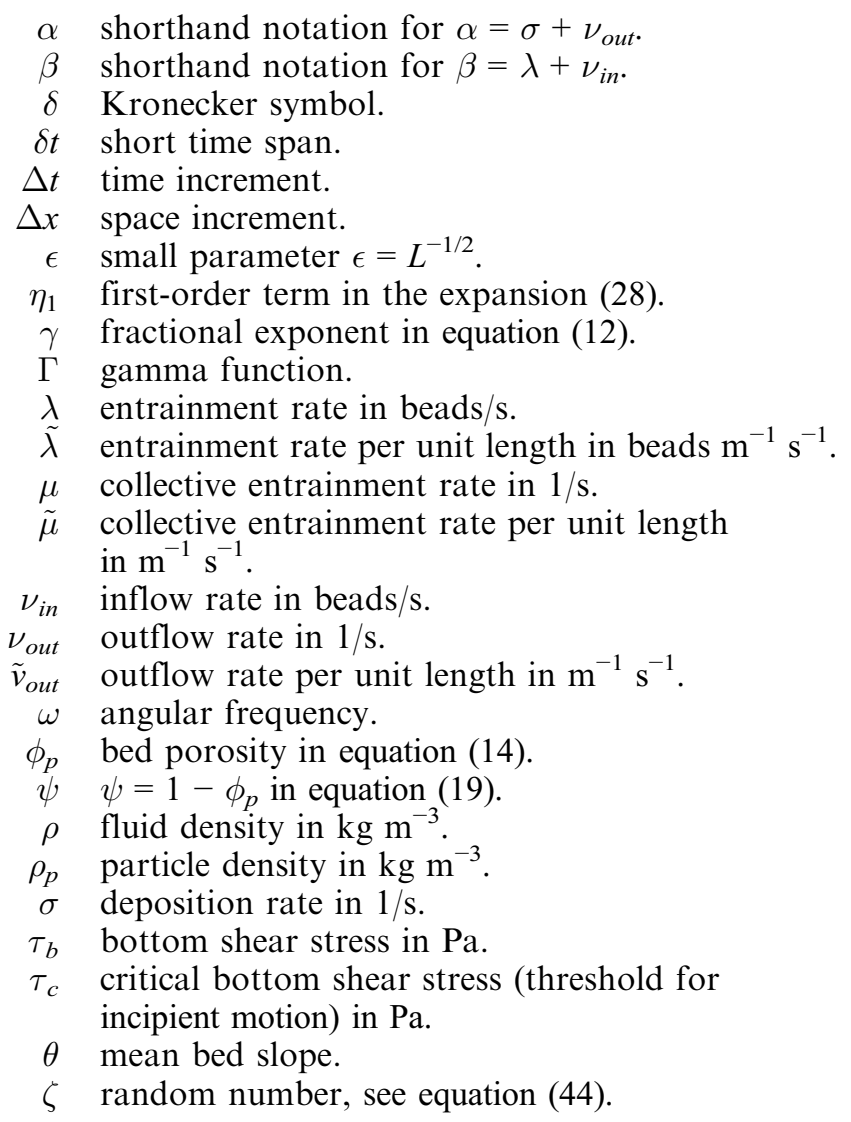

[72] Acknowledgments. The work presented here was supported by the competence centers in Mobile Information and Communication Systems (a center supported by the Swiss National Science Foundation under grant 5005-67322) and in Environmental Sciences (CCES project called Apunch). The author would also like to thank National Center for Earth-surface Dynamics (an NSF Science and Technology Center at the University of Minnesota funded under agreement EAR-0120914) and the Water Cycle Dynamics in a Changing Environment hydrologic synthesis project (University of Illinois, funded under agreement EAR-0636043) for cosponsoring the Stochastic Transport and Emerging Scaling on Earth's Surface working group meeting (Lake Tahoe, November 2007) that fostered the research presented here. I am grateful to the anonymous reviewers and the editorial team for their constructive remarks and incentives, which were essential in revising the manuscript.

\section{References}

Ancey, C., T. Böhm, M. Jodeau, and P. Frey (2006), Statistical description of sediment transport experiments, Phys. Rev. E, 74, 011302.

Ancey, C., A. C. Davison, T. Böhm, M. Jodeau, and P. Frey (2008), Entrainment and motion of coarse particles in a shallow water stream down a steep slope, J. Fluid Mech., 595, 83-114.

Balmforth, N. J., and A. Provenzale (2001), Patterns of dirt, in Geomorphological Fluid Mechanics, edited by N. J. Balmforth and A. Provenzale, pp. 369-393, Springer, Berlin.

Bänziger, R., and H. Burch (1991), Geschiebetransport in Wildbächen: Messung mittels eines neuartigen Sensors (Bedload transport in mountain streams: Measurements through a new sensor system), Schweizer Ing. Archit., 109, 576-579. 
Berkowitz, B., A. Cortis, M. Dentz, and H. Scher (2006), Modeling nonFickian transport in geological formations as a continuous time random walk, Rev. Geophys., 44, RG2003, doi:10.1029/2005RG000178.

Best, J. (2005), The fluid dynamics of river dunes: A review and some future research directions, J. Geophys. Res., 110, F04S02, doi:10.1029/ 2004JF000218.

Blom, A., and G. Parker (2004), Vertical sorting and the morphodynamics of bed form-dominated rivers: A modeling framework, J. Geophys. Res., 109, F02007, doi:10.1029/2003JF000069.

Blom, A., G. Parker, J. S. Ribberink, and H. J. de Vriend (2006), Vertical sorting and the morphodynamics of bed-form-dominated rivers: An equilibrium sorting model, J. Geophys. Res., 111, F01006, doi:10.1029/ 2004JF000175.

Blom, A., J. S. Ribberink, and G. Parker (2008), Vertical sorting and the morphodynamics of bed form-dominated rivers: A sorting evolution model, J. Geophys. Res., 113, F01019, doi:10.1029/2006JF000618.

Böhm, T., C. Ancey, P. Frey, J.-L. Reboud, and C. Duccotet (2004), Fluctuations of the solid discharge of gravity-driven particle flows in a turbulent stream, Phys. Rev. E, 69, 061307.

Böhm, T., P. Frey, C. Duccotet, C. Ancey, M. Jodeau, and J.-L. Reboud (2006), Two-dimensional motion of a set of particles in a free surface flow with image processing, Exp. Fluids, 41, 1-11.

Bridge, J. S., and D. F. Dominic (1984), Bed load grain velocities and sediment transport rates, Water Resour. Res., 20, 476-490.

Bunte, K., and S. R. Abt (2005), Effect of sampling time on measured gravel bed load transport rates in a coarse-bedded stream, Water Resour. Res., 41, W11405, doi:10.1029/2004WR003880.

Carey, W. P. (1985), Variability in measured bedload-transport rates, Water Resour. Bull., 21, 39-48.

Carling, P. A., J. J. Williams, A. Kelsey, M. S. Glaister, and H. G. Orr (1998), Coarse bedload transport in a mountain river, Earth Surf. Processes Landforms, 23, 23-40.

Coleman, S. E., and B. W. Melville (1996), Initiation of bed forms on a flat sand bed, J. Hydraul. Eng., 122, 301-310.

Coleman, S. E., and V. I. Nikora (2009), Bed and flow dynamics leading to sediment-wave initiation, Water Resour. Res., 45, W04402, doi:10.1029/ 2007WR006741.

Coleman, S. E., M. H. Zhang, and T. M. Clunie (2005), Sediment-wave development in subcritical water flow, J. Hydraul. Eng., 131, 106-111.

Cox, D. R., and H. D. Miller (1965), The Theory of Stochastic Processes, Chapman and Hall, Boca Raton, Fla.

Cudden, J. R., and T. B. Hoey (2003), The causes of bedload pulses in a gravel channel: The implications of bedload grain-size distributions, Earth Surf. Processes Landforms, 28, 1411-1428.

DeVries, P. (2002), Bedload layer thickness and disturbance depth in gravel bed streams, J. Hydraul. Eng., 128, 983-991.

Drake, T. G., R. L. Shreve, W. E. Dietrich, and L. B. Leopold (1988), Bedload transport of fine gravel observed by motion-picture photography, J. Fluid Mech., 192, 193-217.

Drew, D. A., and S. L. Passman (1999), Theory of Multicomponent Fluids, Springer, New York.

Einstein, H. A. (1936), Der Geschiebetrieb als Wahrscheinlichkeitsproblem (Bed load transport as a probability problem), Verlag Rascher, Zurich, Switzerland. (English translation, Sedimentation Symposium, edited by H. W. Shen, translated by W. W. Sayre, pp. C1-C105, Colo. State Univ., Fort Collins, 1972.)

Einstein, H. A. (1950), The bed-load function for sediment transportation in open channel flows, Tech. Rep. 1026, U.S. Dep. of Agric., Washington, D. C.

Ergenzinger, P. (1988), The nature of coarse material bed load transport, in Sediment Budgets: Proceedings of a Symposium Held at Porto Alegre, edited by M. P. Bordas and D. E. Walling, IAHS Publ., 174, 207-216.

Exner, F. M. (1925), Über die Wechselwirkung zwischen Wasser und Geschiebe in Flüssen (On the interaction between water and sediment in streams), Sitzungsber. Akad. Wiss. Wien Math. Naturwiss., Abt. 2a, 134, 165-205.

Ferguson, R. I., and T. B. Hoey (2002), Long-term slowdown of river tracer pebbles: Generic models and implications for interpreting short-term tracer studies, Water Resour. Res., 38(8), 1142, doi:10.1029/2001WR000637.

Ferguson, R. I., D. J. Bloomer, T. B. Hoey, and A. Werritty (2002), Mobility of river tracer pebbles over different timescales, Water Resour. Res., 38(5), 1045, doi:10.1029/2001WR000254.

Fernandez Luque, R., and R. van Beek (1976), Erosion and transport of bed-load sediment, J. Hydraul. Res., 14, 127-144.

Frey, P., and J.-L. Reboud (2001), Experimental study of narrow freesurface turbulent flows on steep slopes, in Advances in Flow Modeling and Turbulence Measurements, edited by H. Ninokata, A. Wada, and N. Tanaka, World Sci., Singapore.
Furbish, D. J., S. D. Thorne, T. C. Byrd, J. Warburton, J. J. Cudney, and R. W. Handel (1998), Irregular bed forms in steep, rough channels: 2. Field observations, Water Resour. Res., 34, 3649-3659.

Ganti, V., A. Singh, P. Passalacqua, and E. Foufoula-Georgiou (2009), A subordinated Brownian motion model for sediment transport, Phys. Rev. E, 80,011111 .

Gardiner, C. W. (1983), Handbook of Stochastic Methods, Springer, Berlin.

Gillespie, D. T. (1992), Markov Processes: An Introduction for Physical Scientists, Academic, San Diego, Calif.

Gillespie, D. T. (2007), Stochastic simulation of chemical kinetics, Annu. Rev. Phys. Chem., 58, 35-55.

Gomez, B. (1991), Bedload transport, Earth. Sci. Rev., 31, 89-132.

Graf, W. H. (1984), Hydraulics of Sediment Transport, Water Resour., Littleton, Colo.

Habersack, H. M. (2001), Radio-tracking gravel particles in a large braided river in New Zealand: A field test of the stochastic theory of bed load transport proposed by Einstein, Hydrol. Processes, 15, 377-391.

Hassan, M. A., and M. Church (1991), Distance of movement of coarse particles in gravel bed streams, Water Resour. Res., 27, 503-511.

Heimsath, A. M., D. J. Furbisch, and W. E. Dietrich (2005), The illusion of diffusion: Field evidence for depth-dependent sediment transport, Geology, 33, 949-952.

Herczynski, R., and I. Pienkowska (1980), Toward a statistical theory of suspension, Annu. Rev. Fluid Mech., 12, 237-269.

Higham, D. J. (2001), An algorithmic introduction to numerical simulation of stochastic differential equations, SIAM Rev., 43, 525-546.

Hubbell, D. W., and W. W. Sayre (1964), Sand transport studies with radioactive tracers, J. Hydraul. Div. Am. Soc. Civ. Eng., 90, 39-68.

Iacus, S. M. (2008), Simulation and Inference for Stochastic Differential Equations, Springer, New York.

Jerolmack, D. J., and D. Mohrig (2005), A unified model for subaqueous bed form dynamics, Water Resour. Res., 41, W12421, doi:10.1029/ 2005 WR004329.

Julien, P.-Y. (1994), Erosion and Sedimentation, Cambridge Univ. Press, Cambridge, U. K.

Kovacs, A., and G. Parker (1994), A new vectorial bedload formulation and its application to the time evolution of straight rivers, J. Fluid Mech., 267, 153-183.

Kubatko, E. J., and J. J. Westerink (2007), Exact discontinuous solutions of Exner's bed evolution model: Simple theory for sediment bores, J. Hydraul. Eng., 133, 305-311.

Kuhnle, R. A., and J. B. Southard (1988), Bed load transport fluctuations in a gravel bed laboratory channel, Water Resour. Res., 24, 247-260.

Kuhnle, R. A., and J. C. Willis (1998), Statistics of sediment transport in Goodwin Creek, J. Hydraul. Eng., 124, 1109-1114.

Lhuillier, D. (1992), Ensemble averaging in slightly non-uniform suspensions, Eur. J. Mech. B, 6, 649-661.

Marchioro, M., M. Tanksley, and A. Prosperetti (2000), Flow of spatially non-uniform suspensions: Part I: Phenomenology, Int. J. Multiphase Flow, 26, 783-831.

McNamara, J. P., and C. Borden (2004), Observations on the movement of coarse gravel using implanted motion-sensing radio transmitters, Hydrol. Processes, 18, 1871-1884.

Metzler, R., and J. Klafter (2004), The restaurant at the end of the random walk: Recent developments in the description of anomalous transport by fractional dynamics, J. Phys. A Math. Gen., 37, R161-R208.

Nakagawa, H., and T. Tsujimoto (1980), Sand bed instability due to bed load motion, J. Hydraul. Eng., 106, 2029-2051.

Nakagawa, H., and T. Tsujimoto (1984), Spectral analysis of sand bed instability, J. Hydraul. Eng., 110, 467-483.

Nikora, V. I., A. N. Sukhodolov, and P. M. Rowinski (1997), Statistical sand wave dynamics in one-directional water flows, J. Fluid Mech., $351,17-39$.

Nikora, V. I., D. G. Goring, and B. J. F. Biggs (1998), On gravel-bed roughness characterization, Water Resour. Res., 34, 517-527.

Nikora, V., H. Habersack, T. Huber, and I. McEwan (2002), On bed particle diffusion in gravel bed flows under weak bed load transport, Water Resour. Res., 38(6), 1081, doi:10.1029/2001WR000513.

Niño, Y., A. Atala, M. Barahona, and D. Aracena (2002), Discrete particle model for analyzing bedform development, J. Hydraul. Eng., 128, 381389.

Paola, C., P. L. Heller, and C. L. Angevine (1992), The large-scale dynamics of grain-size variation in alluvial basins, 1: Theory, Basin Res., 4, 73-90.

Parker, G., C. Paola, and S. Leclair (2000), Probabilistic Exner sediment continuity equation for mixtures with no active layer, J. Hydraul. Eng., $126,818-826$.

Postma, G., M. G. Kleinhans, P. T. Meijer, and J. T. Eggenhuisen (2008), Sediment transport in analogue flume models compared with real-world 
sedimentary systems: A new look at scaling evolution of sedimentary systems in a flume, Sedimentology, 55, 1541-1557.

Pryce, R. S., and P. E. Ashmore (2003), The relation between particle path length distributions and channel morphology in gravel-bed streams: A synthesis, Geomorphology, 1353, 1-21.

Radice, A. (2009), Use of the Lorenz curve to quantify statistical nonuniformity of sediment transport rate, J. Hydraul. Eng., 135, 320-326.

Rickenmann, D., and B. W. McArdell (2007), Continuous measurement of sediment transport in the Erlenbach stream using piezoelectric bedload impact sensors, Earth Surf. Processes Landforms, 32, 1362-1378.

Rickenmann, D., and B. McArdell (2008), Calibration of piezoelectric bedload impact sensors in the Pitzbach mountain stream, Geodin. Acta, 21(1-2), 35-52.

Rümelin, W. (1982), Numerical treatment of stochastic differential equations, SIAM J. Numer. Anal., 19, 604-613.

Schmeeckle, M. W., J. M. Nelson, and R. L. Shreve (2007), Forces on stationary particles in near-bed turbulent flows, J. Geophys. Res., 112, F02003, doi:10.1029/2006JF000536.

Schmidt, K.-H., and P. Ergenziger (1992), Bedload entrainment, travel lengths, step lengths, rest periods: Studied with passive (iron, magnetic) and active (radio) tracer techniques, Earth Surf. Processes Landforms, 17, 147-165.

Schumer, R., M. M. Meerschaert, and B. Baeumer (2009), Fractional advection-dispersion equations for modeling transport at the Earth surface, J. Geophys. Res., 114, F00A07, doi:10.1029/2008JF001246.

Seminara, G., L. Solari, and G. Parker (2002), Bed load at low Shields stress on arbitrarily sloping beds: Failure of the Bagnold hypothesis, Water Resour. Res., 38(11), 1249, doi:10.1029/2001WR000681.

Singh, A., K. Fienberg, D. J. Jerolmack, J. Marr, and E. Foufoula-Georgiou (2009), Experimental evidence for statistical scaling and intermittency in sediment transport rates, J. Geophys. Res., 114, F01025, doi:10.1029/ 2007JF000963.

Sornette, D. (2000), Critical Phenomena in Natural Sciences, Springer, New York.

Stark, C. P., E. Foufoula-Georgiou, and V. Ganti (2009), A nonlocal theory of sediment buffering and bedrock channel evolution, J. Geophys. Res., 114, F01029, doi:10.1029/2008JF000981.
Tipper, J. C. (2007), The 'stochastic river': The use of budget-capacity modelling as a basis for predicting long-term properties of stratigraphic successions, Sediment. Geol., 202, 269-280.

Tucker, G. E., and D. N. Bradley (2010), Trouble with diffusion: Reassessing hillslope erosion laws with a particle-based model, J. Geophys. Res. 115, F00A10, doi:10.1029/2009JF001264

Tunnicliffe, J., A. S. Gottesfeld, and M. Mohamed (2000), High resolution measurement of bedload transport, Hydrol. Processes, 14, 2631-2643.

Turcotte, D. L. (1995), Scaling in geology: Landforms and earthquakes, Proc. Natl. Acad. Sci. U. S. A., 92, 6697-6704.

Turowski, J. M., A. Badoux, D. Rickenmann, and B. Fritschi (2008), Erfassung des Sedimenttransportes in Wildbächen und Gerbigsflüssen: Anwendungsmöglichkeiten von Geophonmessanlagen (Recording bedload transport in torrents and mountain streams: Potential application with geophone-based systems), Wasser Energ. Luft, 100, 69-74.

van Rijn, L. (1984), Sediment transport, part III: Bed forms and alluvial roughness, J. Hydraul. Eng., 110, 1733-1754.

Voller, V. R., and C. Paola (2010), Can anomalous diffusion describe depositional fluvial profiles?, J. Geophys. Res., doi:10.1029/2009JF001278, in press.

Wiberg, P. L., and J. D. Smith (1989), Model for calculating bedload transport of sediment, J. Hydraul. Eng., 115, 101-123.

Wilcock, P. R. (1997), Entrainment, displacement and transport of tracer gravels, Earth Surf. Processes Landforms, 22, 1125-1138.

Wong, M., G. Parker, P. DeVries, T. M. Brown, and S. J. Burges (2007), Experiments on dispersion of tracer stones under lower-regime plane-bed equilibrium bed load transport, Water Resour. Res., 43, W03440, doi:10.1029/2006WR005172.

Yalin, M. S. (1963), An expression for bed-load transportation, J. Hydraul. Div. Am. Soc. Civ. Eng., 89, 221-249.

Yalin, M. S. (1992), River Mechanics, Pergamon, Oxford, U. K.

C. Ancey, School of Architecture, Civil and Environmental Engineering, École Polytechnique Fédérale de Lausanne, CH-1015 Lausanne, Switzerland. (christophe.ancey@epfl.ch) 